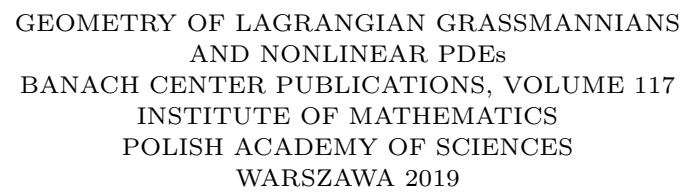

\title{
SURFACES IN LAGUERRE GEOMETRY
}

\author{
EMILIO MUSSO \\ Dipartimento di Scienze Matematiche, Politecnico di Torino \\ Corso Duca degli Abruzzi 24, I-10129 Torino, Italy \\ E-mail: emilio.musso@polito.it
}

LORENZO NICOLODI

Dipartimento di Scienze Matematiche, Fisiche e Informatiche, Università di Parma Parco Area delle Scienze 53/A, I-43124 Parma, Italy

E-mail: lorenzo.nicolodi@unipr.it

\begin{abstract}
This exposition gives an introduction to the theory of surfaces in Laguerre geometry and surveys some significant results concerning three important classes of surfaces in Laguerre geometry, namely $L$-isothermic, $L$-minimal, and generalized $L$-minimal surfaces. The quadric model of Lie sphere geometry is adopted for Laguerre geometry and the method of moving frames is used throughout. The Cartan-Kähler theorem for exterior differential systems is applied to study the Cauchy problem for the Pfaffian differential system of $L$-minimal surfaces. This paper is an elaboration of our talks at the IMPAN Workshop in Warsaw. Our objective was to illustrate, by the subject of Laguerre surface geometry, some of the main concepts presented in the lecture series by G. R. Jensen on Lie sphere geometry and by B. McKay on exterior differential systems.
\end{abstract}

1. Introduction. Laguerre geometry is a classical sphere geometry that has its origins in the work of E. Laguerre in the mid 19th century and that had been extensively studied in the 1920s by Blaschke and Thomsen [6, 7]. The study of surfaces in Laguerre geometry is currently still an active area of research [3, 37, 38, 42, 43, 44, 47, 49, 53, 55, 54, and several classical topics in Laguerre geometry, such as Laguerre minimal surfaces and Laguerre isothermic surfaces and their transformation theory, have recently received much

2010 Mathematics Subject Classification: Primary 53A35; Secondary 53C42.

Key words and phrases: Laguerre geometry, Lie sphere geometry, surfaces in Laguerre geometry, Legendre immersions, Laguerre Gauss map, l-minimal surfaces, $L$-isothermic surfaces, $L$-applicable surfaces, Lawson correspondence.

The paper is in final form and no version of it will be published elsewhere. 
attention in the theory of integrable systems [46, 47, 57, 63, 60, in discrete differential geometry, and in the applications to geometric computing and architectural geometry [8, 10, 9, 59, 58, 61.

The fundamental elements of Laguerre geometry in Euclidean space $\mathbb{R}^{3}$ are oriented planes, oriented spheres and points. The orientation is determined by a field of unit normals and the basic relation among these elements is that of oriented contact. Laguerre geometry distinguishes between oriented planes and oriented spheres (including point spheres). To describe oriented planes, oriented spheres and points in a uniform way we will use the quadric model of Lie sphere geometry [7, 21, 31, 33. In this model, all oriented planes, oriented spheres and point spheres in $\mathbb{R}^{3} \cup\{\infty\}$ are in bijective correspondence with the points on a quadric $\mathcal{Q}$ in real projective space $\mathbb{R P}^{5}$ given by the equation $\langle\mathfrak{a}, \mathfrak{a}\rangle=0$, where $\langle$,$\rangle is a nondegenerate scalar product of signature (4,2)$ in $\mathbb{R}^{6}$. Oriented contact is determined by the conjugacy relation with respect to $\mathcal{Q}$. The 5 -dimensional space $\boldsymbol{\Lambda}$ of lines in $\mathcal{Q}$ which do not meet the "point at infinity" $\{\infty\}$ is the underlying space for Laguerre geometry. The transformations of Laguerre geometry are bijective, separately, on the set $\mathcal{Q}_{\Pi}$ of oriented planes and the set $\mathcal{Q}_{\Sigma}$ of oriented spheres (including point spheres) in $\mathbb{R}^{3}$, fix the "point at infinity" and preserve oriented contact. In the quadric model, the group of Laguerre transformations is a subgroup of the group of Lie sphere transformations, which leave invariant the space of all oriented spheres, oriented planes and point spheres in $\mathbb{R}^{3} \cup\{\infty\}$. The Laguerre group is isomorphic to the 10-dimensional (restricted) Poincaré group [7, 21.

An immersed surface $x: S \rightarrow \mathbb{R}^{3}$, oriented by a unit normal field $n: S \rightarrow S^{2}$, induces a Legendre immersion $f=(x, n): S \rightarrow \mathbb{R}^{3} \times S^{2}=T_{1} \mathbb{R}^{3}$ into the unit tangent bundle of $\mathbb{R}^{3}$, endowed with the natural contact structure given by the one-form $\alpha_{(x, n)}=d x \cdot n$. The space $\mathbb{R}^{3} \times S^{2}$ can be identified with $\boldsymbol{\Lambda}$ as contact manifold. The Laguerre space is $\boldsymbol{\Lambda} \cong \mathbb{R}^{3} \times S^{2}$ viewed as homogeneous space of the Laguerre group. The Laguerre group acts on the Legendre lifts of the immersions rather than on the immersions themselves, since it does not act by point-transformations. The principal aim of Laguerre geometry is to study the properties of an immersion which are invariant under the action of the Laguerre group on Legendre immersions. Locally and up to Laguerre transformation, any Legendre immersion arises as a Legendre lift. Thus, the study of Laguerre geometry of surfaces in $\mathbb{R}^{3}$ is reduced to that of Legendre immersions in $\Lambda \cong \mathbb{R}^{3} \times S^{2}$. The most important tool to study the Laguerre geometry of surfaces in $\mathbb{R}^{3}$ is the Laguerre position vector $\mathfrak{s}: S \rightarrow \mathbb{R}^{6},\langle\mathfrak{s}, \mathfrak{s}\rangle=0$, which corresponds to the classical middle sphere congruence, also known as the Laguerre Gauss map. For a given immersion $x: S \rightarrow \mathbb{R}^{3}$, by pulling back $\langle$,$\rangle via \mathfrak{s}$ we obtain a Laguerre invariant metric on $S$. This metric is conformal to the third fundamental form III $=d n \cdot d n$ of the immersion $x$.

The purpose of this article is to survey some significant results concerning three important classes of surfaces in Laguerre geometry, namely, $L$-isothermic, $L$-minimal, and generalized $L$-minimal surfaces. These are the Laguerre geometric counterparts of isothermic, Willmore, and generalized Willmore surfaces in Möbius geometry (cf. Remark 7.3). Traditionally, see the work of Blaschke and Thomsen [7], Laguerre and Möbius surface geometries have been developed in parallel as subgeometries of Lie sphere geometry.

In Section 2, we recall the basic facts about Laguerre geometry in Euclidean space 
using the quadric model of Lie sphere geometry.

In Section 3 , we develop the method of moving frames for Legendre immersions in the Laguerre space $\boldsymbol{\Lambda}$ and show how to construct a canonical frame field for a Legendre immersion under the hypothesis of nondegeneracy 1$]$

In Section 4 , we discuss $L$-isothermic surfaces. A surface in $\mathbb{R}^{3}$ is $L$-isothermic if, away from umbilic and parabolic points, it admits curvature line coordinates that are isothermal (conformal) with respect to the third fundamental form [7, 44, 46]. $L$-isothermic surfaces are invariant under the Laguerre group and are characterized as the only $L$-applicable surfaces $2^{2}$ Moreover, the nontrivial family of surfaces that are $L$-applicable on a given $L$-isothermic surface depends on one parameter. This feature indicates that $L$-isothermic surfaces constitute an integrable system. For more details on the aspects of $L$-isothermic surfaces related to the theory of integrable system and for the study of their rich transformation theory, including the analogues of the $T$-transformation and of the Darboux transformation in Möbius geometry, we refer the reader to [45, 44, 46, 47, 49, 53, 63, 60.

In Section 5, we give an account of the theory of $L$-minimal surfaces. A smooth immersed surface in $\mathbb{R}^{3}$ with no parabolic points is called Laguerre minimal (L-minimal) if it is an extremal of the Weingarten functional

$$
\int\left(H^{2} / K-1\right) d A
$$

where $H$ and $K$ are the mean and Gauss curvatures of the immersion, and $d A$ is the induced area element of the surface [6, 7, 43, 55. The functional and so its critical points are preserved by the Laguerre group. For a Legendre immersion $f=(x, n): S \rightarrow \boldsymbol{\Lambda}$, the Laguerre Gauss map is the map $\sigma_{f}: S \rightarrow \mathcal{Q}_{\Sigma}$ which assigns to each $s \in S$ the middle sphere, that is, the oriented sphere of $\mathbb{R}^{3}$ of radius $H / K$ which is in oriented contact with the tangent plane of $x$ at $x(s)$. Away from umbilics and parabolic points, it turns out that $\sigma_{f}$ is a spacelike immersion into $\mathcal{Q}_{\Sigma}$, which is isometric to Minkowski 4 -space $\mathbb{R}^{3,1}$. Actually, $\sigma_{f}$ is a marginally outer trapped surface (MOTS) in $\mathbb{R}^{3,1}$ (cf. [52, 53] and the literature therein). Interestingly enough, one can prove that $\sigma_{f}$ has zero mean curvature vector in $\mathcal{Q}_{\Sigma} \cong \mathbb{R}^{3,1}$ if and only if $f$ is $L$-minimal [7, 43. Associated to an arbitrary nondegenerate Legendre surface there is a naturally defined quartic differential $\mathfrak{Q}_{f}$ and a quadratic differential $\mathfrak{P}_{f}$ (cf. Section 7). An interesting feature of $L$-minimal immersions is that this quartic differential is holomorphic [43].

In Section 6, $L$-minimal surfaces are interpreted as integral manifolds of a suitable quasi-linear Pfaffian differential system on the manifold $M=L \times \mathbb{R}^{4}$. The basic techniques of the theory of exterior differential systems are applied to study the Cauchy problem for this system. We first use Cartan's test to prove that the system is in involution and its solutions depend on four functions in one variable. Then, we compute the polar equations of the system and apply the Cartan-Kähler theorem to solve the Cauchy problem.

In Section 7, nondegenerate Legendre immersions $f$ whose associate quartic differential $\mathfrak{Q}_{f}$ is holomorphic are considered. Such immersions are called generalized L-minimal. We prove that a nondegenerate Legendre immersion $f: S \rightarrow \boldsymbol{\Lambda}$ is generalized $L$-minimal

\footnotetext{
${ }^{1}$ cf. Section 3 . Definition 3.3

${ }^{2}$ cf. Section $\overline{4}$. Definition $\overline{4.4}$
} 
if and only if the immersion is $L$-minimal (in which case $\mathfrak{P}_{f}$ is zero), or is locally the $T$-transform of an $L$-minimal isothermic surface. Using this, $L$-minimal isothermic surfaces and their $T$-transforms can be characterized in terms of the differential geometry of their Laguerre Gauss maps. It is proved that a Legendre immersion $f: S \rightarrow \boldsymbol{\Lambda}$ is $L$-minimal and $L$-isothermic if and only if its Laguerre Gauss map $\sigma_{f}: S \rightarrow \mathbb{R}^{3,1}$ has zero mean curvature in some spacelike, timelike, or (degenerate) isotropic hyperplane of $\mathbb{R}^{3,1}$. Moreover, $f$ is generalized $L$-minimal with non-zero $\mathfrak{P}_{f}$ if and only if its Laguerre Gauss map $\sigma_{f}: S \rightarrow \mathbb{R}^{3,1}$ has constant mean curvature $H=r$ in some translate of hyperbolic 3 -space $\mathbb{H}^{3}\left(-r^{2}\right) \subset \mathbb{R}^{3,1}$, de Sitter 3 -space $\mathbb{S}_{1}^{3}\left(r^{2}\right) \subset \mathbb{R}^{3,1}$, or has zero mean curvature in some translate of a time-oriented lightcone $\mathcal{L}_{ \pm}^{3} \subset \mathbb{R}^{3,1}$. If the Laguerre Gauss map of $f$ takes values in a spacelike (respectively, timelike, isotropic) hyperplane, then the Laguerre Gauss maps of the $T$-transforms of $f$ take values in a translate of a hyperbolic 3-space (respectively, de Sitter 3-space, time-oriented lightcone). As an application of these results (cf. [53]), various instances of the Lawson isometric correspondence [35] between certain isometric constant mean curvature (CMC) surfaces in different hyperbolic 3-spaces and of the generalizations of Lawson's correspondence in the Lorentzian [54] and the (degenerate) isotropic situations [1, 3, 34, 36], can be viewed as special cases of the $T$-transformation of $L$-isothermic surfaces with holomorphic quartic differential.

For the subject of exterior differential systems and the Cartan-Kähler theorem we refer the reader to the monographs [15, 20, 24, 25, 29] and [40. The summation convention over repeated indices is used throughout the paper.

\section{Preliminaries and definitions}

2.1. Notation. Vectors in $\mathbb{R}^{6}$ are denoted by $\mathfrak{a}, \mathfrak{b}, \ldots$, and the components of a vector $\mathfrak{a}$ with respect to the standard basis $\boldsymbol{\epsilon}_{0}, \ldots, \boldsymbol{\epsilon}_{5}$ are indicated by $\mathfrak{a}^{j}, j=0, \ldots, 5$. Vectors in $\mathbb{R}^{4}$ are denoted by capital letters $V, W, \ldots$, and the components of a vector $V$ with respect to the standard basis $e_{0}, \ldots, e_{3}$ are indicated by $V^{a}, a=0, \ldots, 3$. Vectors of $\mathbb{R}^{3}$ are denoted by $u, v, \ldots$, and the components of a vector $v$ with respect to the standard basis are indicated by $v^{i}, i=1,2,3$.

For $X \in \mathfrak{g l}(3, \mathbb{R})$ and $B \in \mathfrak{g l}(4, \mathbb{R})$, we let

$$
\widehat{X}=\left[\begin{array}{ll}
1 & 0 \\
0 & X
\end{array}\right] \in \mathfrak{g l}(4, \mathbb{R}), \quad \widetilde{B}=K B K^{-1} \in \mathfrak{g l}(4, \mathbb{R}),
$$

where

$$
K=\left[\begin{array}{cccc}
\frac{1}{\sqrt{2}} & \frac{1}{\sqrt{2}} & 0 & 0 \\
0 & 0 & 1 & 0 \\
0 & 0 & 0 & 1 \\
\frac{1}{\sqrt{2}} & -\frac{1}{\sqrt{2}} & 0 & 0
\end{array}\right], \quad K^{-1}=\left[\begin{array}{cccc}
\frac{1}{\sqrt{2}} & 0 & 0 & \frac{1}{\sqrt{2}} \\
\frac{1}{\sqrt{2}} & 0 & 0 & -\frac{1}{\sqrt{2}} \\
0 & 1 & 0 & 0 \\
0 & 0 & 1 & 0
\end{array}\right] .
$$

For $v \in \mathbb{R}^{3}$ and $V \in \mathbb{R}^{4}$, we let $\widehat{v}, \widetilde{V}$ and $\mathfrak{a}(V)$ be given by

$$
\widehat{v}={ }^{t}(0, v) \in \mathbb{R}^{4}, \quad \widetilde{V}=K V, \quad \mathfrak{a}(V)={ }^{t}\left(1, \tilde{V}, \frac{(V, V)}{2}\right),
$$

where $\left(\right.$, ) denotes the Lorentz scalar product of $\mathbb{R}^{4}$ given by

$$
(V, W)=-V^{0} W^{0}+V^{1} W^{1}+V^{2} W^{2}+V^{3} W^{3} .
$$


We let $\mathbb{R}^{3,1}$ denote $\mathbb{R}^{4}$ with the above Lorentz scalar product.

2.2. The Laguerre group. Let $\mathbb{R}^{4,2}$ denote $\mathbb{R}^{6}$ with the orientation induced by the standard basis $\boldsymbol{\epsilon}_{0}, \ldots, \boldsymbol{\epsilon}_{5}$ and the scalar product of signature $(4,2)$ given by

$$
\langle\mathfrak{a}, \mathfrak{b}\rangle=-\left(\mathfrak{a}^{0} \mathfrak{b}^{5}+\mathfrak{a}^{5} \mathfrak{b}^{0}\right)-\left(\mathfrak{a}^{1} \mathfrak{b}^{4}+\mathfrak{a}^{4} \mathfrak{b}^{1}\right)+\mathfrak{a}^{2} \mathfrak{b}^{2}+\mathfrak{a}^{3} \mathfrak{b}^{3}={ }^{t} \mathfrak{a} \eta \mathfrak{b}
$$

where

$$
\eta=\left(\eta_{i j}\right)=\left[\begin{array}{ccc}
0 & 0 & -L \\
0 & I_{2} & 0 \\
-L & 0 & 0
\end{array}\right], \quad L=\left[\begin{array}{ll}
0 & 1 \\
1 & 0
\end{array}\right], \quad I_{2}=\left[\begin{array}{ll}
1 & 0 \\
0 & 1
\end{array}\right]
$$

Let $G$ be the identity component of the pseudo-orthogonal group $\mathrm{O}(\eta)$ of 2.1 ,

$$
\mathrm{O}(\eta)=\left\{{ }^{\prime} A \in \mathrm{GL}(6, \mathbb{R}):{ }^{t} A \eta A=\eta\right\} .
$$

The Laguerre group $L$ is the subgroup of $G$ consisting of all $A$ for which $A\left(\epsilon_{5}\right)=\epsilon_{5}$, i.e.,

$$
L:=\left\{A \in G: A\left(\boldsymbol{\epsilon}_{5}\right)=\boldsymbol{\epsilon}_{5}\right\} .
$$

In other words, $L$ is the 10-dimensional connected Lie group consisting of all unimodular $6 \times 6$ matrices $A$ satisfying the conditions

$$
\langle A \mathbf{x}, A \mathbf{y}\rangle=\langle\mathbf{x}, \mathbf{y}\rangle, \quad-\left\langle A\left(\boldsymbol{\epsilon}_{1}+\boldsymbol{\epsilon}_{4}\right), \boldsymbol{\epsilon}_{1}+\boldsymbol{\epsilon}_{4}\right\rangle \geq 2, \quad A\left(\boldsymbol{\epsilon}_{5}\right)=\boldsymbol{\epsilon}_{5} .
$$

The Lie algebra of $L$ is

$$
\mathfrak{l}=\left\{a=\left(a_{i}^{j}\right) \in \mathfrak{g l}(6, \mathbb{R}): \eta_{i k} a_{j}^{k}+\eta_{j k} a_{i}^{k}=0 ; a_{5}^{j}=0\right\} .
$$

2.2.1. Lorentz transformations, rotations, and boosts. Let $G_{6}$ denote the group of restricted Lorentz transformations of $\mathbb{R}^{3,1}$ and $P_{10}=G_{6} \rtimes \mathbb{R}^{4}$ the corresponding inhomogeneous group, the restricted Poincaré group. Retaining the notation of $\$ 2.1$, every $A \in L$ can be written in the form

$$
A(B ; V)=\left[\begin{array}{ccc}
1 & 0 & 0 \\
\widetilde{V} & \widetilde{B} & 0 \\
\frac{(V, V)}{2} & * \widetilde{V} \widetilde{B} & 1
\end{array}\right],
$$

where $V \in \mathbb{R}^{4}, B \in G_{6}$, and for each $W={ }^{t}\left(W^{0}, W^{1}, W^{2}, W^{3}\right) \in \mathbb{R}^{4}$,

$$
{ }^{*} \widetilde{V} W=-\left(\widetilde{V}^{0} W^{3}+\widetilde{V}^{3} W^{0}\right)+\widetilde{V}^{1} W^{1}+\widetilde{V}^{2} W^{2} .
$$

The mapping

$$
G_{6} \rtimes \mathbb{R}^{4} \ni(B ; V) \longmapsto A(B ; V) \in L
$$

is an isomorphism of Lie groups, which also gives a representation of the group $\mathbb{E}(3)=$ $\mathrm{SO}(3) \rtimes \mathbb{R}^{3}$ of Euclidean motions into the Laguerre group,

$$
\mathrm{SO}(3) \rtimes \mathbb{R}^{3} \ni(R ; v) \longmapsto A(\widehat{R} ; \widehat{v}) \in L .
$$

The image of 2.4 coincides with the closed subgroup of $L$ consisting of all elements $A$ fixing the vector $\boldsymbol{\epsilon}_{1}+\boldsymbol{\epsilon}_{4}$. More explicitly, for $(R ; v) \in \mathrm{SO}(3) \rtimes \mathbb{R}^{3}$, where $R=\left(r_{j}^{i}\right)$ and 
$v={ }^{t}\left(v^{1}, v^{2}, v^{3}\right)$, we compute

$$
A(\widehat{R} ; \widehat{v})=\left[\begin{array}{cccccc}
1 & 0 & 0 & 0 & 0 & 0 \\
\frac{v^{1}}{\sqrt{2}} & \frac{1+r_{1}^{1}}{2} & \frac{r_{2}^{1}}{\sqrt{2}} & \frac{r_{3}^{1}}{\sqrt{2}} & \frac{1-r_{1}^{1}}{2} & 0 \\
v^{2} & \frac{r_{1}^{2}}{\sqrt{2}} & r_{2}^{2} & r_{3}^{2} & \frac{-r_{1}^{2}}{\sqrt{2}} & 0 \\
v^{3} & \frac{r_{1}^{3}}{\sqrt{2}} & r_{2}^{3} & r_{3}^{3} & \frac{-r_{1}^{3}}{\sqrt{2}} & 0 \\
\frac{-v^{1}}{\sqrt{2}} & \frac{1-r_{1}^{1}}{2} & \frac{-r_{2}^{1}}{\sqrt{2}} & \frac{-r_{3}^{1}}{\sqrt{2}} & \frac{1+r_{1}^{1}}{2} & 0 \\
\frac{t v v}{2} & \frac{r_{1}^{i} v^{i}}{\sqrt{2}} & r_{2}^{i} v^{i} & r_{3}^{i} v^{i} & \frac{-r_{1}^{i} v^{i}}{\sqrt{2}} & 1
\end{array}\right] .
$$

Let $B_{b} \in G_{6}$ be the pure Lorentz transformation (pure Lorentz boost) of $\mathbb{R}^{3,1}$ given by the symmetric matrix

$$
B_{b}=\left[\begin{array}{cc}
\beta & -\beta^{t} b \\
-\beta b & I_{3}+\frac{\beta-1}{t b b} b^{t} b
\end{array}\right],
$$

where $b \in \mathbb{R}^{3},{ }^{t} b b<1$, is the relative-velocity vector and $\beta=\left(1-{ }^{t} b b\right)^{-\frac{1}{2}}$. We let $A(b) \in L$ denote the element of the Laguerre group corresponding to $B_{b}$ by means of 2.3 , i.e., $A(b)=A\left(B_{b} ; 0\right)$. Since every restricted Lorentz transformation $B \in G_{6}$ has a unique decomposition as a product of a pure rotation $\widehat{R}, R \in \mathrm{SO}(3)$, followed by a pure Lorentz boost $B_{b}, B=B_{b} \widehat{R}$, it follows that every Laguerre transformation $A \in L$ can be decomposed as a product

$$
A=E A(b) T(s),
$$

where, via 2.3 and (2.4), $E$ corresponds to a Euclidean motion, $A(b)$ corresponds to a pure Lorentz transformation with velocity vector $b \in \mathbb{R}^{3}$, and $T(s)$ corresponds to the time translation $\tau_{s}:^{t}\left(V^{0}, V^{1}, V^{2}, V^{3}\right) \mapsto{ }^{t}\left(V^{0}+s, V^{1}, V^{2}, V^{3}\right)$ of the Poincaré group.

2.3. The structure equations of Laguerre group. For every $A \in L$, let $A_{j}=A \boldsymbol{\epsilon}_{j}$ denote the $j$ th column vector of $A$. Thus, $\left(A_{0}, \ldots, A_{5}\right)$ is a Laguerre frame, i.e., a basis of $\mathbb{R}^{6}$ such that

$$
\left\langle A_{i}, A_{j}\right\rangle=\eta_{i j}, \quad A_{5}=\epsilon_{5}
$$

and $-\left\langle A_{1}+A_{4}, \boldsymbol{\epsilon}_{1}+\boldsymbol{\epsilon}_{4}\right\rangle \geq 2, i, j=0, \ldots, 5$. Regarding the columns $A_{j}, j=0, \ldots, 5$, of $A$ as $\mathbb{R}^{6}$-valued functions, there are unique one-forms $\left\{\omega_{j}^{i}\right\}_{\{i, j=0, \ldots, 5\}}$ so that

$$
d A_{j}=\omega_{j}^{i} A_{i},
$$

where $\omega=\left(\omega_{j}^{i}\right)=A^{-1} d A$ is the the Maurer-Cartan form of $L$, i.e., the left-invariant l-valued one-form $\omega=A^{-1} d A$.

Exterior differentiation of (2.6) and 2.7) yields the structure equations of the Laguerre group:

$$
\begin{aligned}
0 & =\omega_{i}^{k} \eta_{k j}+\omega_{j}^{k} \eta_{k i}, \\
\omega_{5}^{k} & =0, \\
d \omega_{j}^{i} & =-\omega_{k}^{i} \wedge \omega_{j}^{k} .
\end{aligned}
$$


It follows that

$$
\omega=\left(\omega_{j}^{i}\right)=\left[\begin{array}{cccccc}
0 & 0 & 0 & 0 & 0 & 0 \\
\omega_{0}^{1} & \omega_{1}^{1} & \omega_{2}^{1} & \omega_{3}^{1} & 0 & 0 \\
\omega_{0}^{2} & \omega_{1}^{2} & 0 & -\omega_{2}^{3} & \omega_{2}^{1} & 0 \\
\omega_{0}^{3} & \omega_{1}^{3} & \omega_{2}^{3} & 0 & \omega_{3}^{1} & 0 \\
\omega_{0}^{4} & 0 & \omega_{1}^{2} & \omega_{1}^{3} & -\omega_{1}^{1} & 0 \\
0 & -\omega_{0}^{4} & \omega_{0}^{2} & \omega_{0}^{3} & -\omega_{0}^{1} & 0
\end{array}\right] .
$$

The left-invariant $\mathfrak{l}$-valued one-form $\omega$ transforms by right translations according to the rule

$$
R_{A}^{*}(\omega)=A^{-1} \omega A, \quad \forall A \in L .
$$

2.4. Laguerre geometry in Euclidean 3-space: The quadric model. A null line in $\mathbb{R}^{4,2}$ is a one-dimensional subspace $[\mathfrak{a}] \subset \mathbb{R}^{4,2}$ spanned by a vector $\mathfrak{a}$ such that $\langle\mathfrak{a}, \mathfrak{a}\rangle=0$. The space of all null lines gives rise to the quadric $\mathcal{Q}$ in real projective space $\mathbb{R P}^{5}$ defined by the equation

$$
\langle\mathfrak{a}, \mathfrak{a}\rangle=-2 \mathfrak{a}^{0} \mathfrak{a}^{5}-2 \mathfrak{a}^{1} \mathfrak{a}^{4}+\left(\mathfrak{a}^{2}\right)^{2}+\left(\mathfrak{a}^{3}\right)^{2}=0 .
$$

The quadric $\mathcal{Q}$ is called the Lie quadric [21, 30.

The Laguerre group $L$ acts on the left on $\mathcal{Q}$ by $A \cdot[\mathfrak{a}]=[A \mathfrak{a}]$. This action has a fixed point $\left\{\left[\boldsymbol{\epsilon}_{5}\right]\right\}$ (the "point at infinity") and two nontrivial orbits,

$$
\begin{gathered}
\mathcal{Q}_{\Sigma}=\left\{[\mathfrak{a}] \in \mathcal{Q}:\left\langle\mathfrak{a}, \boldsymbol{\epsilon}_{5}\right\rangle \neq 0\right\}, \\
\mathcal{Q}_{\Pi}=\left\{[\mathfrak{b}] \in \mathcal{Q}:\left\langle\mathfrak{b}, \boldsymbol{\epsilon}_{5}\right\rangle=0, \mathfrak{b} \wedge \boldsymbol{\epsilon}_{5} \neq 0\right\} .
\end{gathered}
$$

$\mathcal{Q}_{\Sigma}$ is a principal orbit, which is open and dense, while $\mathcal{Q}_{\Pi}$ has dimension 3.

We will now provide a geometric description of the orbits $\mathcal{Q}_{\Sigma}$ and $\mathcal{Q}_{\Pi}$.

2.4.1. The space of oriented spheres. The orientation of a two-sphere in Euclidean space $\mathbb{R}^{3}$ is determined by specifying a field of unit normals: inward normals if the radius is positive, outward normals if the radius is negative. An oriented sphere with center $p$ and signed radius $r$ will be denoted by $S_{r}(p)$. We allow $r=0$, in which case the sphere represents the point $p$; point spheres are not oriented. An oriented sphere $S_{r}(p), p={ }^{t}\left(p^{1}, p^{2}, p^{3}\right)$, can be represented, alternatively, by a vector $V(r, p)={ }^{t}\left(r, p^{1}, p^{2}, p^{3}\right)$ of $\mathbb{R}^{4}$ or by a null line $[\mathfrak{a}]$, spanned by

$$
\mathfrak{a}(r, p)={ }^{t}\left(1, \frac{r+p^{1}}{\sqrt{2}}, p^{2}, p^{3}, \frac{r-p^{1}}{\sqrt{2}}, \frac{{ }^{t} p p-r^{2}}{2}\right) .
$$

These two representations of oriented spheres are equivalent in that they are related by the mapping

$$
\mathbb{R}^{4} \ni V \longmapsto[\mathfrak{a}(V)] \in \mathcal{Q}_{\Sigma}
$$

which is an equivariant diffeomorphism with respect of the action of the (restricted) Poincaré group on $\mathbb{R}^{4}$ and of the Laguerre Group $L$ on $\mathcal{Q}_{\Sigma}$, taking into account the Lie group isomorphism defined by 2.3 . The orbit $\mathcal{Q}_{\Sigma}$ may thus be identified with Minkowski 4 -space $\mathbb{R}^{3,1}$ acted on by the (restricted) Poincarè group.

In particular, point spheres are represented by the elements of

$$
\mathcal{Q}_{\Sigma}^{\prime}=\left\{[\mathfrak{a}] \in \mathcal{Q}_{\Sigma}:\left\langle\mathfrak{a}, \boldsymbol{\epsilon}_{1}+\boldsymbol{\epsilon}_{4}\right\rangle=0\right\}
$$


and proper oriented spheres by the elements of

$$
\mathcal{Q}_{\Sigma}^{\prime \prime}=\left\{[\mathfrak{a}] \in \mathcal{Q}_{\Sigma}:\left\langle\mathfrak{a}, \boldsymbol{\epsilon}_{1}+\boldsymbol{\epsilon}_{4}\right\rangle \neq 0\right\} .
$$

These two subsets are orbits for the action of the Euclidean group $\mathrm{SO}(3) \rtimes \mathbb{R}^{3}$, but are not preserved by the action of the full Laguerre group. A Euclidean motion $(R ; v) \in$ $\mathrm{SO}(3) \rtimes \mathbb{R}^{3}$ maps the sphere $S_{r}(p)$ into the sphere $S_{r}\left(p^{\prime}\right)$ with the same radius $r$ and center $p^{\prime}=R p+v$. A time translation $\tau_{s}$ maps the sphere $S_{r}(p)$ into the sphere $S_{r^{\prime}}(p)$ with the same center $p$ and signed radius $r^{\prime}=r+s$. Finally, a pure Lorentz transformation $B_{b}, b \in \mathbb{R}^{3},{ }^{t} b b<1$, takes the sphere $S_{r}(p)$ to the sphere $S_{r^{\prime}}\left(p^{\prime}\right)$, where

$$
r^{\prime}=\beta\left(r-{ }^{t} b p\right), \quad p^{\prime}=p+\left((\beta-1) \frac{{ }^{t} p b}{t^{b} b}-\beta r\right) b .
$$

2.4.2. The space of oriented planes. Next, we examine the three-dimensional orbit $\mathcal{Q}_{\Pi}$. The orientation of a two-plane $\pi \subset \mathbb{R}^{3}$ is given by fixing a unit normal vector. For $h \in \mathbb{R}$, $p \in \mathbb{R}^{3}$, and $n={ }^{t}\left(n^{1}, n^{2}, n^{3}\right) \in S^{2}$, let $\Pi_{h}(n)$ and $\Pi_{p}(n)$ denote, respectively, the oriented plane with unit normal $n$ and height $h$ given by

$$
\Pi_{h}(n)=\left\{x \in \mathbb{R}^{3}:{ }^{t} n x=h\right\},
$$

and the oriented plane through the point $p$ normal to $n$ given by

$$
\Pi_{p}(n)=\left\{x \in \mathbb{R}^{3}:{ }^{t} n(x-p)=0\right\} .
$$

The oriented plane $\Pi_{p}(n)$ can be uniquely represented by the null line $[\mathfrak{b}(n, p)]$ spanned by the

$$
\mathfrak{b}(n, p)={ }^{t}\left(0, \frac{1+n^{1}}{2}, \frac{n^{2}}{\sqrt{2}}, \frac{n^{3}}{\sqrt{2}}, \frac{1-n^{1}}{2}, \frac{n p}{\sqrt{2}}\right) .
$$

Thus the points of $\mathcal{Q}_{\Pi}$ represent the oriented planes of Euclidean space.

2.4.3. Oriented contact. Two oriented planes $\Pi_{h}(n)$ and $\Pi_{h^{\prime}}\left(n^{\prime}\right)$ are in oriented contact if they have the same unit normals, i.e., $n=n^{\prime}$. Two oriented spheres $S_{r}(p)$ and $S_{r^{\prime}}\left(p^{\prime}\right)$ are in oriented contact if the Euclidean distance $d\left(p, p^{\prime}\right)$ between $p$ and $p^{\prime}$ coincides with $\left|r-r^{\prime}\right|$, that is, $d\left(p, p^{\prime}\right)=\left|r-r^{\prime}\right|$. This amounts to saying that the Lorentz scalar product $\left(V(r, p)-V\left(r^{\prime}, p^{\prime}\right), V(r, p)-V\left(r^{\prime}, p^{\prime}\right)\right)=0$. The sphere $S_{r}(p)$ and the plane $\Pi_{h}(n)$ are in oriented contact if $n^{1} p^{1}+n^{2} p^{2}+n^{3} p^{3}=h+r$. In particular, a point sphere $S_{0}(p)$ and an oriented plane $\Pi_{h}(n)$ are in oriented contact if $p \in \Pi_{h}(n)$.

From the above identifications, it follows that two null lines $[\mathfrak{a}]$ and $[\mathfrak{b}]$ represent oriented spheres or oriented planes in oriented contact if and only if $\langle\mathfrak{a}, \mathfrak{b}\rangle=0$.

2.5. The Laguerre space. We let $\boldsymbol{\Lambda}$ denote the set of all two-dimensional subspaces $\lambda(\mathfrak{a}, \mathfrak{b})$ of $\mathbb{R}^{6}$ spanned by vectors $\mathfrak{a}$ and $\mathfrak{b}$ satisfying

$$
\langle\mathfrak{a}, \mathfrak{a}\rangle=\langle\mathfrak{a}, \mathfrak{b}\rangle=\langle\mathfrak{b}, \mathfrak{b}\rangle=0, \quad \epsilon_{5} \notin \lambda(\mathfrak{a}, \mathfrak{b}) .
$$

We call $\boldsymbol{\Lambda}$ the Laguerre space. This is a 5-dimensional orbit of the action of the Laguerre group $L$ on the Grassmannian $G_{2}(6)$ of two-planes in $\mathbb{R}^{6}$, where the action is given by

$$
A \lambda(\mathfrak{a}, \mathfrak{b}):=\lambda(A \mathfrak{a}, A \mathfrak{b}), \quad \forall A \in L, \forall \mathfrak{a}, \mathfrak{b} \in \mathbb{R}^{6} .
$$

REMARK 2.1. The Laguerre space $\boldsymbol{\Lambda}$ can be seen as the space of projective lines in the Lie quadric $\mathcal{Q}=\{[\mathfrak{a}]:\langle\mathfrak{a}, \mathfrak{a}\rangle=0\}$ which do not meet the "point at infinity" $\left\{\left[\boldsymbol{\epsilon}_{5}\right]\right\}$. The 
Laguerre space $\boldsymbol{\Lambda}$ is a dense open set of the set of all lines in $\mathcal{Q}$. It is the complement of the set of lines through $\left[\boldsymbol{\epsilon}_{5}\right]$ in $\mathcal{Q}$ (cf. [30]) $!^{3}$

We will provide two geometrical realizations of the Laguerre space $\boldsymbol{\Lambda}$.

2.5.1. The Laguerre space as the unit tangent bundle of $\mathbb{R}^{3}$. The Laguerre space $\boldsymbol{\Lambda}$ is identified with the bundle $T_{1}\left(\mathbb{R}^{3}\right) \cong \mathbb{R}^{3} \times S^{2}$ of unit tangent vectors of $\mathbb{R}^{3}$ by means of the map

$$
\mathbb{R}^{3} \times S^{2} \ni(p, n) \longmapsto \lambda(\mathfrak{a}(p), \mathfrak{b}(n, p)) \in \boldsymbol{\Lambda},
$$

where $\mathfrak{a}(p)=\mathfrak{a}(0, p)$ and $\mathfrak{b}(n, p)$ are given, respectively, as in 2.11 and 2.13.

2.5.2. The Laguerre space as the set of parabolic pencils of $\mathbb{R}^{3}$. Let $\Pi_{h}(n)$ be an oriented plane and let $p$ be a point on this plane. The set $\widehat{\lambda}(p, n)$ of all oriented spheres in oriented contact with $\Pi_{h}(n)$ at $p$ is called the parabolic pencil of spheres with base locus $p$ and radical plane $\Pi_{h}(n)$.

If $\hat{\lambda}(p, n)$ is a parabolic pencil with base locus $p$ and radical plane $\Pi_{h}(n)$, the null vectors $\mathfrak{a}(0, p)$ and $\mathfrak{b}(n, p)$ (cf. 2.11) and (2.13) ) span a null plane and the oriented spheres of the pencil are represented by the null lines lying on the null plane spanned by $\mathfrak{a}$ and $\mathfrak{b}$. The Laguerre space can thus be interpreted as the set of all parabolic pencils of oriented spheres in $\mathbb{R}^{3}$.

Given a null plane $\lambda(\mathfrak{a}, \mathfrak{b}) \in \boldsymbol{\Lambda}$, there is a unique $[\hat{\mathfrak{a}}] \subset \lambda(\mathfrak{a}, \mathfrak{b})$ so that $\left\langle\hat{\mathfrak{a}}, \boldsymbol{\epsilon}_{1}+\boldsymbol{\epsilon}_{4}\right\rangle=0$. Thus $[\hat{\mathfrak{a}}]$ represents a point $p(\mathfrak{a}, \mathfrak{b}) \in \mathbb{R}^{3}$, which we call the Euclidean projection of $\lambda(\mathfrak{a}, \mathfrak{b})$. Using 2.14, it follows that the Euclidean projection $p(\mathfrak{a}, \mathfrak{b})$ coincides with the first component in $(p, n)$, where $(p, n)$ is the contact element which represents the null plane $\lambda(\mathfrak{a}, \mathfrak{b})$.

In other words, the Euclidean projection $\Lambda \rightarrow \mathbb{R}^{3}, \lambda \mapsto p(\lambda)$ agrees with the bundle projection map $\mathbb{R}^{3} \times S^{2} \rightarrow \mathbb{R}^{3}$. Observe, however, that the Laguerre group $L$ does not preserve the Euclidean projection.

2.6. The structure equations of the Laguerre space. The Laguerre group $L$ acts transitively on $\boldsymbol{\Lambda}$ and the map

$$
\pi_{L}: L \rightarrow \boldsymbol{\Lambda}, \quad A \longmapsto \lambda\left(A_{0}, A_{1}\right)=A \lambda\left(\boldsymbol{\epsilon}_{0}, \boldsymbol{\epsilon}_{1}\right)
$$

makes $L$ into a principal bundle over $\boldsymbol{\Lambda}$ with structure group

$$
L_{0}=\left\{A=\left(A_{j}^{i}\right) \in L: A_{0}^{j}=A_{1}^{j}=0, j=2,3,4,5\right\} .
$$

The elements of $L_{0}$ are matrices of the form

$$
X(d ; b ; x)=\left[\begin{array}{cccccc}
1 & 0 & 0 & 0 & 0 & 0 \\
d_{1} & d_{2} & \tilde{x}^{1} & \tilde{x}^{2} & \frac{d_{2}}{2}{ }^{t} x x & 0 \\
0 & 0 & b_{1}^{1} & b_{2}^{1} & x^{1} & 0 \\
0 & 0 & b_{1}^{2} & b_{2}^{2} & x^{2} & 0 \\
0 & 0 & 0 & 0 & 1 / d_{2} & 0 \\
0 & 0 & 0 & 0 & -d_{1} / d_{2} & 1
\end{array}\right],
$$

\footnotetext{
${ }^{3}$ Notice that in the lecture notes of G. R. Jensen 31, the symbol $\boldsymbol{\Lambda}$ is used to denote the set of all lines in $\mathcal{Q}$, while the set of lines in $\mathcal{Q}$ which do not meet the point at infinity is denoted by $\boldsymbol{\Lambda}^{\prime}$.
} 
where $b=\left(b_{j}^{i}\right) \in \mathrm{SO}(2), d=\left(d_{1}, d_{2}\right), d_{2} \neq 0, x={ }^{t}\left(x^{1}, x^{2}\right) \in \mathbb{R}^{2},\left(\tilde{x}^{1}, \tilde{x}^{2}\right)=d_{2}{ }^{t} x b$, and $\frac{1}{d_{2}}\left(1+d_{2}^{2}\right)+\frac{1}{2} d_{2}{ }^{t} x x \geq 2$.

A local Laguerre frame field is a local cross section $A: U \subset \boldsymbol{\Lambda} \rightarrow L$ of the Laguerre fibration $\pi_{L}$, where $U$ is an open subset $U$ of $\Lambda$. If $A: U \rightarrow L$ is a Laguerre frame field, any other Laguerre frame field $\hat{A}$ on $U$ is given by

$$
\hat{A}=A X(d ; b ; x),
$$

where $X(d ; b ; x): U \rightarrow L_{0}$ is a smooth map. If we set

$$
\theta=\left(\theta_{j}^{i}\right), \quad \theta_{j}^{i}=A^{*}\left(\omega_{j}^{i}\right),
$$

then the one-forms

$$
\theta_{0}^{2}, \theta_{0}^{3}, \theta_{0}^{4}, \theta_{1}^{2}, \theta_{1}^{3}
$$

define a local coframe on the open subset $U \subset \boldsymbol{\Lambda}$. If $A$ and $\hat{A}$ are related by (2.17), the corresponding $\mathfrak{l}$-valued one-forms $\theta$ and $\hat{\theta}$ are related by

$$
\hat{\theta}=X^{-1} \theta X+X^{-1} d X .
$$

In particular, the coframe 2.18 is subject to the following transformation rules

$$
\left[\begin{array}{c}
\hat{\theta}_{0}^{2} \\
\hat{\theta}_{0}^{3} \\
\hat{\theta}_{0}^{4} \\
\hat{\theta}_{1}^{2} \\
\hat{\theta}_{1}^{3}
\end{array}\right]=\left[\begin{array}{c|c|c}
{ }^{t} b & -d_{2}{ }^{t} b x & d_{1}{ }^{t} b \\
\hline 0 & d_{2} & 0 \\
\hline 0 & 0 & d_{2}{ }^{t} b
\end{array}\right]\left[\begin{array}{c}
\theta_{0}^{2} \\
\theta_{0}^{3} \\
\theta_{0}^{4} \\
\theta_{1}^{2} \\
\theta_{1}^{3}
\end{array}\right] .
$$

According to the transformation rules 2.20, there are three naturally defined subbundles of the tangent bundle $T(\boldsymbol{\Lambda})$ of the Laguerre space:

1. the subbundle $\mathcal{Z} \subset T(\boldsymbol{\Lambda})$, defined by requiring that $\theta_{0}^{4}=0$;

2. the subbundle $\mathcal{H} \subset T(\boldsymbol{\Lambda})$, defined by requiring that $\theta_{1}^{2}=\theta_{1}^{3}=0$;

3. the subbundle $\mathcal{K} \subset T(\boldsymbol{\Lambda})$, defined by requiring that $\theta_{0}^{4}=\theta_{1}^{2}=\theta_{1}^{3}=0$.

Notice that $\operatorname{rank}(\mathcal{Z})=4, \operatorname{rank}(\mathcal{H})=3, \operatorname{rank}(\mathcal{K})=2$, and that $\mathcal{K}=\mathcal{Z} \cap \mathcal{H}$. From the structure equations 2.10 , it follows that

$$
d \theta_{0}^{4} \equiv \theta_{0}^{2} \wedge \theta_{1}^{2}+\theta_{0}^{3} \wedge \theta_{1}^{3} \bmod \left\{\theta_{0}^{4}\right\} .
$$

This implies that $d \theta_{0}^{4} \wedge d \theta_{0}^{4} \wedge \theta_{0}^{4}$ is never zero on $\boldsymbol{\Lambda}$, and hence $\theta_{0}^{4}$ defines a contact structure on the Laguerre space $\boldsymbol{\Lambda}$. The subbundle $\mathcal{Z}$ is a contact distribution on $\boldsymbol{\Lambda}$.

REMARK 2.2. Observe that the one-form $\theta_{0}^{4}=-\left\langle d A_{0}, A_{1}\right\rangle$. Therefore, the one-form $\theta_{0}^{4}$ corresponds via the map 2.14 to the one-form $\frac{1}{\sqrt{2}} \sum_{i=1}^{3} n^{i} d p^{i}=\frac{1}{\sqrt{2}} d p \cdot n$. Thus, the contact structure defined by $\theta_{0}^{4}$ coincides with the natural contact structure on $\mathbb{R}^{3} \times S^{2}$ given by the one-form $\alpha_{(p, n)}=\sum_{i=1}^{3} n^{i} d p^{i}=d p \cdot n$.

\section{Moving frames for Legendre immersions}

Definition 3.1. Let $S$ be an oriented, connected, two-dimensional manifold. A smooth immersion $f: S \rightarrow \boldsymbol{\Lambda}$ is called Legendre if

$$
d f_{\mid s}\left(T_{s} S\right) \subset \mathcal{Z}_{f(s)}, \quad \forall s \in S .
$$

This amounts to saying that $f^{*}\left(\theta_{0}^{4}\right)=0$ on $S$. 
Two Legendre immersions $f, f^{\prime}: S \rightarrow \boldsymbol{\Lambda}$ are said to be $L$-equivalent if there exists $A \in L$, such that $A f^{\prime}(S)=f(S)$. In this case, the two immersions $f$ and $f^{\prime}$ are considered to be the same geometric object.

REMARK 3.2. An immersed surface $x: S \rightarrow \mathbb{R}^{3}$, oriented by a unit normal field $n$ : $S \rightarrow S^{2}$, induces a lift $f=(x, n): S \rightarrow \boldsymbol{\Lambda}$ of $x$ to $\boldsymbol{\Lambda} \cong \mathbb{R}^{3} \times S^{2}$ which is a Legendre immersion, since $d x \cdot n=0$. We call $f$ the Legendre lift of $x$. Locally and up to $L$-equivalence, any Legendre immersion arises in this way. However, observe that in general, if $f=(x, n): S \rightarrow \boldsymbol{\Lambda} \cong \mathbb{R}^{3} \times S^{2}$ is a Legendre immersion, $x$ need not be an immersion into $\mathbb{R}^{3}$. In particular, two immersions $x, x^{\prime}: S \rightarrow \mathbb{R}^{3}$ are $L$-equivalent if their Legendre lifts are $L$-equivalent.

For a generic Legendre immersion $f=(x, n)$, the quadratic form $d n \cdot d n$ is positive semidefinite, that is $d n \cdot d n \geq 0$.

Definition 3.3. A Legendre immersion $f=(x, n): S \rightarrow \boldsymbol{\Lambda}$ is said to be nondegenerate if (1) the quadratic form $d n \cdot d n$ is positive definite and (2) the quadratic forms $d x \cdot d n$ and $d n \cdot d n$ are everywhere linearly independent on $S$.

If $f=(x, n)$ is the Legendre lift of an immersion $x: S \rightarrow \mathbb{R}^{3}$, the condition that $f$ is nondegenerate means that $x$ is umbilic free and its Gauss curvature is everywhere different from zero.

The condition that $d n \cdot d n$ is positive definite will be assumed throughout.

3.1. Construction of the canonical frame. Let $f: S \rightarrow \boldsymbol{\Lambda}$ be a Legendre immersion. A local Laguerre frame field along $f$ is a smooth map $A: U \subset S \rightarrow L$ defined on an open subset $U$ of $S$ such that $\pi_{L}([A(s)])=f(s)$, for every $s \in U$. If $f: S \rightarrow \boldsymbol{\Lambda}$ is a Legendre immersion and $A: U \rightarrow L$ is a Laguerre frame field on $U$, then

$$
\alpha_{0}^{4}=0, \quad \alpha_{1}^{2} \wedge \alpha_{1}^{3} \neq 0
$$

where $\alpha_{j}^{i}:=A^{*}\left(\omega_{j}^{i}\right)$. Differentiating $\alpha_{0}^{4}=0$ and using 2.10 yields

$$
\alpha_{0}^{2} \wedge \alpha_{1}^{2}+\alpha_{0}^{3} \wedge \alpha_{1}^{3}=0
$$

By Cartan's Lemma, there are smooth functions $h_{i j}=h_{j i}: U \rightarrow \mathbb{R}, i, j=1$, , which depend on $A$, such that

$$
\begin{aligned}
& \alpha_{0}^{2}=h_{11} \alpha_{1}^{2}+h_{12} \alpha_{1}^{3} \\
& \alpha_{0}^{3}=h_{12} \alpha_{1}^{2}+h_{22} \alpha_{1}^{3} .
\end{aligned}
$$

Any other frame field $\hat{A}$ on $U$ is given by

$$
\hat{A}=A X(d ; b ; x),
$$

where $X=X(d ; b ; x): U \subset S \rightarrow L_{0}$ is a smooth map. By 2.19 , $\hat{\alpha}$ and $\alpha$ are related by

$$
\hat{\alpha}=X^{-1} \alpha X+X^{-1} d X \text {. }
$$

This implies

$$
\left[\begin{array}{c}
\hat{\alpha}_{1}^{2} \\
\hat{\alpha}_{1}^{3}
\end{array}\right]=d_{2}{ }^{t} b\left[\begin{array}{c}
\alpha_{1}^{2} \\
\alpha_{1}^{3}
\end{array}\right],
$$


and

$$
\left[\begin{array}{c}
\hat{\alpha}_{0}^{2} \\
\hat{\alpha}_{0}^{3}
\end{array}\right]={ }^{t} b\left(\left[\begin{array}{c}
\alpha_{0}^{2} \\
\alpha_{0}^{3}
\end{array}\right]+d_{1}\left[\begin{array}{c}
\alpha_{1}^{2} \\
\alpha_{1}^{3}
\end{array}\right]\right) .
$$

From $(3.2),(3.3)$, and $(3.4)$, it follows that

$$
\left[\begin{array}{ll}
\hat{h}_{11} & \hat{h}_{12} \\
\hat{h}_{12} & \hat{h}_{22}
\end{array}\right]=\frac{1}{d_{2}} b_{b}\left[\begin{array}{cc}
h_{11}+d_{1} & h_{12} \\
h_{12} & h_{22}+d_{1}
\end{array}\right] b .
$$

Therefore,

$$
\left(\hat{h}_{11}+\hat{h}_{22}\right)=\frac{1}{d_{2}}\left[\left(h_{11}+h_{22}\right)+2 d_{1}\right] .
$$

This shows that locally there exist Laguerre frame fields such that

$$
h_{11}+h_{22}=0 .
$$

Definition 3.4. A Laguerre frame field $A: U \rightarrow L$ along $f$ is said to be of first order if $h_{11}+h_{22}=0$ on $U$.

It follows from (3.6) that if $\hat{A}$ and $A$ are first order frame fields on $U$, then $\hat{A}=$ $A X(d ; b ; x)$ and $d_{1}=0$. This means that the totality of first order frame fields defines an $L_{1}$-fiber bundle on $S$ whose structure group is

$$
L_{1}=\left\{X(d ; b ; x) \in L_{0}: d_{1}=0\right\} .
$$

REMARK 3.5. A sphere congruence is a two-parameter family of oriented spheres, i.e., a smooth map $\sigma: S \rightarrow \mathcal{Q}_{\Sigma}$ of a connected surface into the space of oriented spheres. By an envelope of $\sigma: S \rightarrow \mathcal{Q}_{\Sigma}$ we mean a Legendre map $f=(x, n): S \rightarrow \boldsymbol{\Lambda}$ such that $\sigma(s)$ and the plane $\Pi_{x(s)}(n(s))$ are in oriented contact at $x(s)$, for every $s \in S$.

Definition 3.6. Let $f: S \rightarrow \boldsymbol{\Lambda}$ be a Legendre immersion and let $A: U \subset S \rightarrow L$ be a first order frame field along $f$. The smooth map $U \ni s \mapsto\left[A_{0}(s)\right] \in \mathcal{Q}_{\Sigma}$ is independent of the choice of first order frame field $A$. This allows the definition of a smooth map on the whole $S$,

$$
\sigma_{f}: S \rightarrow \mathcal{Q}_{\Sigma}
$$

The map $\sigma_{f}$ determines a sphere congruence which is known as the middle congruence (cf. [7]) or Laguerre Gauss map of $f$. By construction, the Legendre immersion $f: S \rightarrow \boldsymbol{\Lambda}$ is an envelope of the middle congruence.

According to (3.4), for a change of first order frames $\hat{A}=A X$, where $X: U \rightarrow L_{1}$, we have

$$
\left[\begin{array}{c}
\hat{\alpha}_{0}^{2} \\
\hat{\alpha}_{0}^{3}
\end{array}\right]={ }^{t} b\left[\begin{array}{l}
\alpha_{0}^{2} \\
\alpha_{0}^{3}
\end{array}\right] .
$$

This implies that the Legendre immersion $f$ induces on $S$ a quadratic form

$$
\Phi_{f}=\left(\alpha_{0}^{2}\right)^{2}+\left(\alpha_{0}^{3}\right)^{2},
$$

the Laguerre metric of the immersion, and an exterior differential two-form

$$
\Omega_{f}=\alpha_{0}^{2} \wedge \alpha_{0}^{3},
$$

the Laguerre area element of the immersion. 
REMARK 3.7. If the Legendre immersion $f: S \rightarrow \boldsymbol{\Lambda}$ is nondegenerate, the form $\Phi_{f}$ is positive definite and $\left.\Omega_{f}\right|_{s} \neq 0$, for every $s \in S$. In particular, note that $\Phi_{f}$ is the metric induced on $S$ by the Laguerre Gauss map $\sigma_{f}$ from the Lorentz product $\langle$,$\rangle on \mathcal{Q}_{\Sigma} \cong \mathbb{R}^{3,1}$, i.e., $\Phi_{f}=\left\langle d \sigma_{f}, d \sigma_{f}\right\rangle$. Thus, if $f$ is nondegenerate, its Laguerre Gauss map $\sigma_{f}$ defines a spacelike immersion of $S$ into Minkowski 4-space $\mathbb{R}^{3,1}$ and $\Omega_{f}$ is the induced area element of $\sigma_{f}: S \rightarrow \mathcal{Q}_{\Sigma}$.

Let $f: S \rightarrow \boldsymbol{\Lambda}$ be a nondegenerate Legendre immersion and let $A: U \subset S \rightarrow L$ be a first order frame field along $f$. Then there exist smooth functions $a_{1}, a_{2}: U \rightarrow \mathbb{R}$ depending on $A$ such that

$$
\alpha_{0}^{1}=a_{1} \alpha_{0}^{2}+a_{2} \alpha_{0}^{3} .
$$

If $\hat{A}=A X(d ; b ; x)$ is any first order frame field on $U$, where $X: U \rightarrow L_{1}$, it follows from 2.19 that

$$
\hat{\alpha}_{0}^{1}=\frac{1}{d_{2}}\left(\alpha_{0}^{1}-d_{2} x^{1} \alpha_{0}^{2}-d_{2} x^{2} \alpha_{0}^{3}\right) .
$$

If we write $\hat{\alpha}_{0}^{1}=\hat{a}_{1} \hat{\alpha}_{0}^{2}+\hat{a}_{2} \hat{\alpha}_{0}^{3}, 3.8$ and 3.10 yield

$$
\left[\begin{array}{l}
\hat{a}_{1} \\
\hat{a}_{2}
\end{array}\right]=\frac{1}{d_{2}} t_{b}\left[\begin{array}{c}
a_{1}-d_{2} x^{1} \\
a_{2}-d_{2} x^{2}
\end{array}\right] .
$$

From the above transformation formula, it follows that, about any point $s \in S$, there exists an open neighborhood $U$ and a Laguerre frame field of first order defined on $U$ for which $a_{1}=a_{2}=0$, i.e., $\alpha_{0}^{1}=0$.

Definition 3.8. A first order Laguerre frame field $A: U \rightarrow L$ along $f$ is said to be of second order if $\alpha_{0}^{1}=0$ on $U$.

It follows from (3.11) that if $\hat{A}$ and $A$ are second order frame fields on $U$, then $\hat{A}=A X(d ; b ; 0)$ and $d_{1}=0$. This means that the totality of second order frame fields generates an $L_{2}$-fiber bundle on $S$ whose structure group is

$$
L_{2}=\left\{X(d ; b ; x) \in L_{1}: x={ }^{t}(0,0)\right\} .
$$

REMARK 3.9 (The Laguerre transform). For a change of second order frames $\hat{A}=A X$, where $X: U \subset S \rightarrow L_{2}$, it is easily seen that the projective lines $\lambda\left(A_{0}, A_{4}\right)$ and $\lambda\left(\hat{A}_{0}, \hat{A}_{4}\right)$ do coincide. Accordingly, we can define a new Legendre immersion (possibly degenerate)

$$
\check{f}: S \rightarrow \boldsymbol{\Lambda}, \quad \check{f}(s):=\lambda\left(A_{0}(s), A_{4}(s)\right), \quad \forall s \in S .
$$

The Legendre immersion $\check{f}$ is called the Laguerre transform of $f$. The Laguerre transform $\check{f}$ is the second envelope of the middle sphere congruence $\sigma_{f}$.

From 3.5 it follows that about any point $s \in S$ there exists an open neighborhood $U$ and a Laguerre frame field of second order defined on $U$ for which $h_{11}=-h_{22}=1$ and $h_{12}=0$.

Definition 3.10. A second order Laguerre frame field $A: U \rightarrow L$ along $f$ for which $h_{11}=-h_{22}=1$ and $h_{12}=0$ on $U$ is called a canonical Laguerre frame field. 
According to 3.5 , two canonical frames $\hat{A}, A: U \rightarrow L$ are related by $\hat{A}=A X(d ; b ; 0)$, where $X$ is a smooth map taking values in the group

$$
\mathbb{Z}_{2}=\left\{X(d ; b ; x) \in L_{2}: d_{2}=1, b= \pm\left[\begin{array}{ll}
1 & 0 \\
0 & 1
\end{array}\right]\right\} .
$$

If $A=\left(A_{0}, A_{1}, A_{2}, A_{3}, A_{4}, A_{5}\right): U \subset S \rightarrow L$ is a canonical frame along $f: S \rightarrow \boldsymbol{\Lambda}$ and $U$ is connected, the only other canonical frame on $U$ is given by

$$
\hat{A}=\left(A_{0}, A_{1},-A_{2},-A_{3}, A_{4}, A_{5}\right) .
$$

A canonical frame $A=\left(A_{0}, A_{1}, A_{2}, A_{3}, A_{4}, A_{5}\right)$ is characterized by the following equations

$$
\left\{\begin{array}{l}
d A_{0}=\alpha_{0}^{2} A_{2}+\alpha_{0}^{3} A_{3}, \\
d A_{1}=\alpha_{1}^{1} A_{1}+\alpha_{0}^{2} A_{2}-\alpha_{0}^{3} A_{3}, \\
d A_{2}=\alpha_{2}^{1} A_{1}+\alpha_{2}^{3} A_{3}+\alpha_{0}^{2}\left(A_{4}+A_{5}\right), \\
d A_{3}=\alpha_{3}^{1} A_{1}-\alpha_{2}^{3} A_{2}+\alpha_{0}^{3}\left(-A_{4}+A_{5}\right), \\
d A_{4}=\alpha_{2}^{1} A_{2}+\alpha_{3}^{1} A_{3}-\alpha_{1}^{1} A_{4}, \\
d A_{5}=0 .
\end{array}\right.
$$

The totality of canonical frame fields on $S$ defines a $\mathbb{Z}_{2}$-fiber bundle $\pi_{f}: \mathcal{F}(f) \rightarrow S$, where

$$
\mathcal{F}(f)=\{(s, A(s)) \in S \times L\}
$$

and $A$ is a canonical frame field along $f$ defined on a neighborhood of $s$, and $\pi_{f}: \mathcal{F}(f) \ni$ $(s, A(s)) \mapsto s \in S$.

REMARK 3.11. Up to $L$-equivalence, and a 2:1 covering, any nondegenerate Legendre immersion of an oriented surface $S$ admits a globally defined canonical frame. In fact, consider a connected component $\tilde{S}$ of $\mathcal{F}(f)$ and let $\tilde{f}: \tilde{S} \rightarrow \boldsymbol{\Lambda}$ be given by $\tilde{f}=f \circ \pi_{f}$. By construction, $\tilde{f}$ is again a nondegenerate Legendre immersion and $f(S)=\tilde{f}(\tilde{S})$. Moreover, $\tilde{A}: \tilde{S} \rightarrow L,(s, A(s)) \mapsto A(s)$, is a global canonical frame along $\tilde{f}: \tilde{S} \rightarrow \boldsymbol{\Lambda}$.

3.2. The structure equations of the canonical frame. Let $f: S \rightarrow \Lambda$ be a nondegenerate Legendre immersion and let $A: S \rightarrow L$ be a canonical frame field along $f$. According to Remark 3.11, it is not restrictive to assume that $A$ globally defined on $S$. From the above discussion, $\left(\alpha_{0}^{2}, \alpha_{0}^{3}\right)$ defines a coframe on $S$, referred to as the canonical coframe, and

$$
\alpha_{1}^{2}=\alpha_{0}^{2}, \quad \alpha_{1}^{3}=-\alpha_{0}^{3}, \quad \alpha_{0}^{1}=0, \quad \alpha_{0}^{4}=0 .
$$

Differentiating $\alpha_{0}^{1}=0$ yields

$$
\alpha_{2}^{1} \wedge \alpha_{0}^{2}+\alpha_{3}^{1} \wedge \alpha_{0}^{3}=0 .
$$

Thus, by Cartan's Lemma there exist smooth functions $p_{1}, p_{2}, p_{3}: S \rightarrow \mathbb{R}$ such that

$$
\alpha_{2}^{1}=p_{1} \alpha_{0}^{2}+p_{2} \alpha_{0}^{3}, \quad \alpha_{3}^{1}=p_{2} \alpha_{0}^{2}+p_{3} \alpha_{0}^{3} .
$$

Next, write

$$
\alpha_{2}^{3}=q_{1} \alpha_{0}^{2}+q_{2} \alpha_{0}^{3},
$$


where $q_{1}, q_{2}: S \rightarrow \mathbb{R}$ are smooth functions. We call the functions $p_{1}, p_{2}, p_{3}, q_{1}, q_{2}$ the invariant functions of the Legendre immersion $f$ (with respect to the canonical frame field $A$ ). Exterior differentiation of $\alpha_{0}^{2}$ and $\alpha_{0}^{3}$, using the structure equations 2.10 and equation (3.16), yields

$$
d \alpha_{0}^{2}=q_{1} \alpha_{0}^{2} \wedge \alpha_{0}^{3}, \quad d \alpha_{0}^{3}=q_{2} \alpha_{0}^{2} \wedge \alpha_{0}^{3} .
$$

Differentiating $\alpha_{1}^{2}$ and $\alpha_{1}^{3}$, using the structure equations 2.10) and the equations (3.17), yields

$$
\alpha_{1}^{1}=2 q_{2} \alpha_{0}^{2}-2 q_{1} \alpha_{0}^{3}
$$

From the structure equations 2.10 , we also obtain the following equations:

$$
\begin{aligned}
& d q_{1} \wedge \alpha_{0}^{2}+d q_{2} \wedge \alpha_{0}^{3}=\left(p_{3}-p_{1}-q_{1}^{2}-q_{2}^{2}\right) \alpha_{0}^{2} \wedge \alpha_{0}^{3}, \\
& d q_{1} \wedge \alpha_{0}^{3}-d q_{2} \wedge \alpha_{0}^{2}=-p_{2} \alpha_{0}^{2} \wedge \alpha_{0}^{3}, \\
& d p_{1} \wedge \alpha_{0}^{2}+d p_{2} \wedge \alpha_{0}^{3}=\left(-3 q_{1} p_{1}-4 q_{2} p_{2}+q_{1} p_{3}\right) \alpha_{0}^{2} \wedge \alpha_{0}^{3}, \\
& d p_{2} \wedge \alpha_{0}^{2}+d p_{3} \wedge \alpha_{0}^{3}=\left(-3 q_{2} p_{3}-4 q_{1} p_{2}+q_{2} p_{1}\right) \alpha_{0}^{2} \wedge \alpha_{0}^{3} .
\end{aligned}
$$

The equations (3.17), 3.19, 3.20), 3.21 and 3.22 will be referred to as the structure equations of the Legendre immersion $f$. The existence of a canonical frame field along $f$ under the nondegeneracy assumption was first proved in [43. The smooth functions $q_{1}$, $q_{2}, p_{1}, p_{2}, p_{3}$ form a complete system of Laguerre invariants for $f$.

REMARK 3.12. If $A: S \rightarrow L$ is a canonical frame field along $f$, the only other canonical frame field is given by $\hat{A}$ as in 3.12 . Under this frame change, the invariants $p_{1}, p_{2}, p_{3}, q_{1}, q_{2}$ transform by

$$
\hat{q}_{1}=-q_{1}, \quad \hat{q}_{2}=-q_{2}, \quad \hat{p}_{1}=p_{1}, \quad \hat{p}_{2}=p_{2}, \quad \hat{p}_{3}=p_{3} .
$$

Thus, there are well defined global functions J, w $: S \rightarrow \mathbb{R}$ such that locally

$$
\mathrm{J}=\frac{1}{2}\left(p_{1}-p_{3}\right), \quad \mathrm{W}=\frac{1}{2}\left(p_{1}+p_{3}\right) .
$$

3.3. Framed Legendre immersions and the Laguerre Pfaffian system. In this section, nondegenerate Legendre immersions will be interpreted as integral manifolds of a quasi-linear Pfaffian differential system.

By a framed Legendre immersion $(S, f, A)$ is meant a nondegenerate Legendre immersion $f: S \rightarrow \Lambda$ endowed with a canonical frame $A: S \rightarrow L$. The immersion $f$ is then given by $\pi_{L} \circ A$ and the normal frame field $A$ satisfies the conditions

$$
\alpha_{0}^{2} \wedge \alpha_{0}^{3} \neq 0, \quad \alpha_{1}^{2}-\alpha_{0}^{2}=\alpha_{1}^{3}+\alpha_{0}^{3}=\alpha_{0}^{1}=\alpha_{0}^{4}=0 .
$$

Conversely, if $A: S \rightarrow L$ is a smooth map satisfying 3.24 , then $f=\pi_{L} \circ A: S \rightarrow \boldsymbol{\Lambda}$ is a nondegenerate Legendre immersion and $A$ is a canonical frame field along $f$. Thus framed Legendre immersions can be interpreted as the integral manifolds of the Pfaffian differential system on $L$ defined by the equations

$$
\omega_{1}^{2}-\omega_{0}^{2}, \quad \omega_{1}^{3}+\omega_{0}^{3}=0, \quad \omega_{0}^{1}=0, \quad \omega_{0}^{4}=0,
$$

with independence condition $\omega_{0}^{2} \wedge \omega_{0}^{3} \neq 0$. This Pfaffian differential system is not in involution. Its involutive prolongation is given by the following system. 
Let $M:=L \times \mathbb{R}^{5}$ and denote by $\left(q_{1}, q_{2}, p_{1}, p_{2}, p_{3}\right)$ the coordinates in $\mathbb{R}^{5}$. Let $\omega^{1}=\omega_{0}^{2}, \omega^{2}=\omega_{0}^{3}$, and define on $M$ the absolute parallelism given by the coframe field $\left(\omega^{i}, \eta^{a}, \pi^{i}, \zeta^{s}\right), a=1, \ldots, 8 ; i=1,2 ; s=1,2,3$, where

$$
\begin{aligned}
& \eta^{1}=\omega_{0}^{4}, \quad \eta^{2}=\omega_{0}^{1}, \quad \eta^{3}=\omega_{1}^{2}-\omega^{1}, \quad \eta^{4}=\omega_{1}^{3}+\omega^{1}, \\
& \eta^{5}=\omega_{2}^{3}-q_{1} \omega^{1}-q_{2} \omega^{2}, \quad \eta^{6}=\omega_{1}^{1}-2 q_{2} \omega^{1}+2 q_{1} \omega^{2} \\
& \eta^{7}=\omega_{2}^{1}-p_{1} \omega^{1}-p_{2} \omega^{2}, \quad \eta^{8}=\omega_{3}^{1}-p_{2} \omega^{1}-p_{3} \omega^{2} \\
& \pi^{i}=d q_{i}, \quad \zeta^{s}=d p_{s} .
\end{aligned}
$$

Let $\mathcal{I}$ be the ideal of the algebra of exterior differential forms on $M$ generated by

$$
\eta^{1}, \ldots, \eta^{8}, d \eta^{1}, \ldots, d \eta^{8} .
$$

Let $(\mathcal{I}, \Omega)$ be the Pfaffian differential system on $M$ defined by the differential ideal $\mathcal{I}$ with the independence condition

$$
\Omega=\omega^{1} \wedge \omega^{2} \neq 0 .
$$

If $\left(A, q_{1}, q_{2}, p_{1}, p_{2}, p_{3}\right): S \rightarrow M$ is an integral manifold of $(\mathcal{I}, \Omega)$, then $f: S \rightarrow \boldsymbol{\Lambda}$, $s \mapsto f(s)=\pi_{L}(A(s))$, defines a Legendre immersion with canonical frame field $A$ and corresponding invariant functions $q_{1}, q_{2}, p_{1}, p_{2}, p_{3}$. Conversely, any framed Legendre immersion $(S, f, A)$ defines an integral manifold of the Pfaffian system $(\mathcal{I}, \Omega)$ by

$$
S \ni s \longmapsto\left(A(s), q_{1}, q_{2}, p_{1}, p_{2}, p_{3}\right) \in M,
$$

where $\left(q_{1}, q_{2}, p_{1}, p_{2}, p_{3}\right)$ are the invariant functions of $A$. Summarizing, framed Legendre immersions, together with the associate invariant functions, may be regarded as being the integral manifolds of the Pfaffian system $(\mathcal{I}, \Omega)$ on $M$.

Definition 3.13. The Pfaffian system $(\mathcal{I}, \Omega)$ on $M$ will be referred to as the Laguerre differential system and will be denoted by $(M, \mathcal{I}, \Omega)$.

3.4. Relations with Euclidean geometry. Let $f=(x, n): S \rightarrow \boldsymbol{\Lambda}$ be the Legendre lift of an oriented immersion $x: S \rightarrow \mathbb{R}^{3}$ with unit normal field $n$ and suppose that $x$ is umbilic free and that its Gauss curvature is everywhere different from zero. We may assume the existence of a global principal frame field $e=\left(n, e_{2}, e_{3} ; x\right): S \rightarrow \mathbb{E}(3)$ along $x$, such that, at every point $s \in S,\left(e_{2}, e_{3}\right)_{\mid s}$ is a positive basis of $d x_{\mid s}\left(T_{s} S\right)$ and $e_{2}, e_{3}$ are along the principal directions. The frame field $e$ satisfies the equations

$$
d x=\varphi^{2} e_{2}+\varphi^{3} e_{3}, \quad d e_{2}=\varphi_{2}^{3} e_{3}-\varphi_{1}^{2} n, \quad d e_{3}=-\varphi_{2}^{3} e_{2}-\varphi_{1}^{3} n,
$$

and

$$
d n=\varphi_{1}^{2} e_{2}+\varphi_{1}^{3} e_{3},
$$

where $\left(\varphi^{2}, \varphi^{3}\right)$ is the dual coframe of $\left(e_{2}, e_{3}\right)$. The condition that $e_{2}, e_{3}$ are along principal directions is expressed by

$$
\varphi_{1}^{2}=-a \varphi^{2}, \quad \varphi_{1}^{2}=-c \varphi^{3},
$$

where $a>c$ are the principal curvatures.

By (2.4) and 2.5), the principal frame field $e$ gives rise to a Laguerre frame field $A=A\left(n, e_{2}, e_{3} ; x\right)$ along the Legendre lift $f=(x, n)$ of the immersion $x$. An easy 
computation shows that the frame field $A$ satisfies the equations

$$
\left\{\begin{array}{l}
d A_{0}=\varphi^{2} A_{2}+\varphi^{3} A_{3} \\
d A_{1}=\frac{\varphi_{1}^{2}}{\sqrt{2}} A_{2}+\frac{\varphi_{1}^{3}}{\sqrt{2}} A_{3} \\
d A_{2}=-\frac{\varphi_{1}^{2}}{\sqrt{2}} A_{1}+\varphi_{2}^{3} A_{3}+\frac{\varphi_{1}^{2}}{\sqrt{2}} A_{4}+\varphi^{2} A_{5} \\
d A_{3}=-\frac{\varphi_{1}^{3}}{\sqrt{2}} A_{1}-\alpha_{2}^{3} A_{2}+\varphi_{1}^{3} A_{4}+\varphi^{3} A_{5} \\
d A_{4}=-\frac{\varphi_{1}^{2}}{\sqrt{2}} A_{2}-\frac{\varphi_{1}^{3}}{\sqrt{2}} A_{3} \\
d A_{5}=0
\end{array}\right.
$$

According to 3.13 , we have

$$
\alpha_{0}^{2}=-\frac{\sqrt{2}}{a} \varphi_{1}^{2}, \quad \alpha_{0}^{3}=-\frac{\sqrt{2}}{c} \varphi_{1}^{3} .
$$

Following the reduction procedure described in $\$ 3.1$, we may adapt $A$ to a first order Laguerre frame field $A^{\prime}$ along the Legendre lift $f$, by setting

$$
A^{\prime}=A X\left(d ; I_{2} ; 0\right), \quad d=\left(\sqrt{2} \frac{H}{K}, 1\right),
$$

where $H=\frac{1}{2}(a+c)$ is the mean curvature and $K=a c$ the Gauss curvature of $x$. The one-form ${\alpha^{\prime}}_{0}^{2}$ and $\alpha_{0}^{\prime 3}$ take the form

$$
\alpha_{0}^{\prime 2}=-\frac{c-a}{2 K} \varphi_{1}^{2}, \quad \alpha_{0}^{\prime 3}=\frac{c-a}{2 K} \varphi_{1}^{3} .
$$

Therefore,

$$
\Phi_{f}=\frac{H^{2}-K}{K^{2}} d n \cdot d n, \quad \Omega_{f}=-\frac{H^{2}-K}{K} d A
$$

where $d A$ denotes the Euclidean area element of $x: S \rightarrow \mathbb{R}^{3}$.

REMARK 3.14. An easy computation shows that, with respect to the first order frame field $A^{\prime}$, the Laguerre Gauss map $\sigma_{f}: S \rightarrow \mathcal{Q}_{\Sigma}$ is expressed by

$$
\sigma_{f}=S_{\frac{H}{K}}\left(x+\frac{H}{K} n\right)
$$

that is, $\sigma_{f}(s)$ represents the oriented sphere centered at $x(s)+\frac{H}{K}(s) n(s)$ with signed radius $\frac{H}{K}(s)$.

If we let

$$
d\left(\frac{H}{K}\right)=\frac{c-a}{2 K}\left(\left(\frac{H}{K}\right)_{2} \varphi_{1}^{2}+\left(\frac{H}{K}\right)_{3} \varphi_{1}^{3}\right)
$$

we compute

$$
\alpha_{0}^{\prime 1}=-\sqrt{2}\left(\frac{H}{K}\right)_{2} \alpha_{0}^{2}+\sqrt{2}\left(\frac{H}{K}\right)_{3} \alpha_{0}^{\prime 3} .
$$

We then further adapt $A^{\prime}$ to a second order frame field $A^{\prime \prime}$ along $f$,

$$
A^{\prime \prime}=A^{\prime} X\left(d ; I_{2} ; y\right), \quad d=(0,1), \quad y={ }^{t}\left(-\sqrt{2}\left(\frac{H}{K}\right)_{2}, \sqrt{2}\left(\frac{H}{K}\right)_{3}\right) .
$$


It turns out that the frame field $A^{\prime \prime}$ is actually a canonical frame field along $f$ and that the corresponding canonical coframe field is given by

$$
\alpha_{0}^{\prime \prime 2}=-\frac{c-a}{2 K} \varphi_{1}^{2}, \quad \alpha_{0}^{\prime \prime 3}=\frac{c-a}{2 K} \varphi_{1}^{3} .
$$

4. L-isothermic surfaces. An important class of surfaces that belong to Laguerre geometry is that of $L$-isothermic surfaces.

Definition 4.1. A nondegenerate Legendre immersion $f: S \rightarrow \boldsymbol{\Lambda}$, with canonical frame field $A$, is called $L$-isothermic if there exist local coordinates which simultaneously diagonalize the definite pair of quadratic forms $\left\langle d A_{0}, d A_{0}\right\rangle=\left(\alpha_{0}^{2}\right)^{2}+\left(\alpha_{0}^{3}\right)^{2}$ and $\left\langle d A_{0}, d A_{1}\right\rangle=\left(\alpha_{0}^{2}\right)^{2}-\left(\alpha_{0}^{3}\right)^{2}$ and which are isothermal with respect to $\left\langle d A_{0}, d A_{0}\right\rangle$.

REMARK 4.2. If $x: S \rightarrow \mathbb{R}^{3}$ is an immersed surface without umbilic and parabolic points, oriented by the unit normal field $n$, the $L$-isothermic condition amounts to the existence of isothermal (conformal) curvature line coordinates for the pair of quadratic forms III $=d n \cdot d n$ and II $=d f \cdot d n$. Examples of $L$-isothermic surfaces include surfaces of revolution, molding surfaces, surfaces with plane lines of curvature [46], minimal surfaces, etc. The notion of $L$-isothermic surfaces is already in Bianchi [5], but the systematic study of $L$-isothermic surfaces was initiated and developed by Blaschke [7].

The following result was proved in 44].

THEOREM 4.3 ([44]). A nondegenerate Legendre immersion $f: S \rightarrow \boldsymbol{\Lambda}$ is L-isothermic if and only if $p_{2}=0$.

We first came across $L$-isothermic surfaces when addressing the question of applicability of surfaces in Laguerre geometry. We recall the following.

Definition 4.4. Let $f, \hat{f}: S \rightarrow \boldsymbol{\Lambda}$ be two nondegenerate Legendre immersions, with respective canonical frame fields $A, \hat{A}$ and canonical coframe fields $\left(\alpha_{0}^{2}, \alpha_{0}^{3}\right),\left(\hat{\alpha}_{0}^{2}, \hat{\alpha}_{0}^{3}\right)$. If $f$ and $\hat{f}$ are not $L$-equivalent, then $f$ and $\hat{f}$ are $L$-applicable on each other if $\alpha_{0}^{2}= \pm \hat{\alpha}_{0}^{2}$, $\alpha_{0}^{3}= \pm \hat{\alpha}_{0}^{3}$. We say that $f: S \rightarrow \boldsymbol{\Lambda}$ is $L$-applicable if, for each $s \in S$, there exists an open neighborhood $U$ of $s$, such that $f_{\mid U}$ is $L$-applicable on some $\hat{f}$.

REMARK 4.5. If $f=(x, n)$ and $\hat{f}=(\hat{x}, \hat{n})$ are the Legendre lifts of two immersions $x: S \rightarrow \mathbb{R}^{3}$ and $\hat{x}: S \rightarrow \mathbb{R}^{3}$, with unit normal fields $n$ and $\hat{n}$, respectively, then $f$ and $\hat{f}$ are $L$-applicable if there exists a (local) orientation-preserving conformal transformation between $(S$, III $=d n \cdot d n)$ and $(S$, III $=d \hat{n} \cdot d \hat{n})$ that preserves the curvature lines of $x$ and $\hat{x}$.

REMARK 4.6. The above definition is related to the general deformation theory of submanifolds in homogeneous spaces as developed by Fubini, Cartan, Griffiths and Jensen (cf. [19, 23, 30]). In fact, it has been proved that two Legendre immersions $f$ and $\hat{f}$ are $L$-applicable if and only if there exists a smooth map $B: S \rightarrow L$ with the property that $B(s) \hat{f}$ and $f$ agree up to order two at $s$, for each $s \in S$. In other words, if and only if $f$ and $\hat{f}$ are second order deformations of each other with respect to the Laguerre group. For the proof of this result and for more details on the deformation problem of surfaces in Laguerre geometry we refer to 44 and [49]. 
The notion of $L$-applicability introduced in Definition 4.4 is the analogue, in Laguerre geometry, of the following notion of applicability in Euclidean geometry: two noncongruent (relative to rigid motions) immersions $x, \hat{x}: S \rightarrow \mathbb{R}^{3}$ are applicable if they are connected by a (local) isometry that preserves the lines of curvature. It is known that the only applicable surfaces in this sense are the molding surfaces $4^{4}$ Actually, such surfaces belong to a one-parameter family of noncongruent surfaces, which are connected by isometries preserving the lines of curvature. For more on molding surfaces and for the proof of this result we refer to [16, §5, Theorem 5.1] or [15, Chapter IV, Theorem 8.1]. The above notion of $L$-applicability is also related to the notion of applicability in Möbius geometry: two noncongruent (relative to Möbius transformations) immersions $x, \hat{x}: S \rightarrow \mathbb{R}^{3} \cup\{\infty\}$ are $M$-applicable if they are connected by a (local) conformal transformation that preserves the lines of curvature. The only $M$-applicable surfaces are the isothermic surfaces $5^{5}$ Also in this case, such surfaces belong to a one-parameter family of noncongruent surfaces, which are connected by conformal transformations preserving the lines of curvature (cf. [41]). For more on isothermic surfaces in Möbius geometry, we refer to [27] and [33].

$L$-applicable surfaces can be characterized in a similar way.

THEOREM 4.7 ([44]). The L-isothermic immersions are the only Legendre immersions that are L-applicable. Moreover, any L-isothermic immersion belongs to a one-parameter family of non-equivalent L-applicable Legendre immersions.

For a given $L$-isothermic immersion, we will now describe the nontrivial one-parameter family of its $L$-applicable immersions. If $f: S \rightarrow \boldsymbol{\Lambda}$ is $L$-isothermic, there are local curvature line coordinates $z=x+i y$ such that the canonical coframe $\left(\alpha_{0}^{2}, \alpha_{0}^{3}\right)$ takes the form

$$
\alpha_{0}^{2}=e^{u} d x, \quad \alpha_{0}^{3}=e^{u} d y,
$$

for a smooth function $u$ on $S$. We call $\Phi=e^{u}$ the Blaschke potential of $f$.

Accordingly, from (3.17), 3.19 and 3.20 it follows that

$$
\begin{gathered}
q_{1}=-e^{-u} u_{y}, \quad q_{2}=e^{-u} u_{x}, \\
p_{1}-p_{3}=-e^{-2 u} \Delta u .
\end{gathered}
$$

Moreover, using (3.21) and 3.22 yields

$$
\begin{aligned}
d\left(e^{2 u}\left(p_{1}+p_{3}\right)\right)= & -e^{2 u}\left\{\left(e^{-2 u} \Delta u\right)_{x}+4 u_{x}\left(e^{-2 u} \Delta u\right)\right\} d x \\
& +e^{2 u}\left\{\left(e^{-2 u} \Delta u\right)_{y}+4 u_{y}\left(e^{-2 u} \Delta u\right)\right\} d y .
\end{aligned}
$$

The integrability condition of 4.3 is the so-called Blaschke equation,

$$
\Delta\left(e^{-u}\left(e^{u}\right)_{x y}\right)=0,
$$

\footnotetext{
${ }^{4}$ We recall (cf. [16, 15]) that a molding surface $S$ can be described kinematically as follows: Take a cylinder $Z$ and a curve $C$ on one of the tangent planes to $Z$. The surface $S$ is the locus described by $C$ as the plane rolls about $Z$. Among the molding surfaces there are the surfaces of revolution.

${ }^{5}$ We recall that a surface is isothermic if it admits conformal curvature line coordinates away from umbilic points.
} 
which can be viewed as the completely integrable (soliton) equation governing $L$-isothermic surfaces (cf. [45, 46]).

Conversely, let $U$ be a simply connected domain in $\mathbb{C}$, and let $\Phi=e^{u}$ be a solution to the Blaschke equation (4.4). It follows that the right hand side of (4.3) is a closed one-form, say $\eta_{\Phi}$. Thus, $\eta_{\Phi}=d \mathcal{K}$, for some function $\mathcal{K}$ determined up to an additive constant. If we let

$$
\mathrm{W}=\mathcal{K} e^{-2 u}, \quad \mathrm{~J}=-\frac{1}{2} e^{-2 u} \Delta u
$$

(cf. (3.23) for the definition of $\mathrm{w}$ and $\mathrm{J}$ ), the one-form defined by

$$
\alpha=\left[\begin{array}{cccccc}
0 & 0 & 0 & 0 & 0 & 0 \\
0 & 2 d u & (\mathrm{~W}+\mathrm{J}) e^{u} d x & (\mathrm{~W}-\mathrm{J}) e^{u} d y & 0 & 0 \\
e^{u} d x & e^{u} d x & 0 & u_{y} d x-u_{x} d y & (\mathrm{~W}+\mathrm{J}) e^{u} d x & 0 \\
e^{u} d y & -e^{u} d y & -u_{y} d x+u_{x} d y & 0 & (\mathrm{~W}-\mathrm{J}) e^{u} d y & 0 \\
0 & 0 & e^{u} d x & -e^{u} d y & -2 d u & 0 \\
0 & 0 & e^{u} d x & e^{u} d y & 0 & 0
\end{array}\right]
$$

satisfies the Maurer-Cartan integrability condition

$$
d \alpha+\alpha \wedge \alpha=0
$$

and then integrates to a map $A=U \rightarrow L$, such that $d A=A \alpha$. The map $f: U \rightarrow \boldsymbol{\Lambda}$ defined by

$$
f=\lambda\left(A_{0}, A_{1}\right)
$$

is a smooth Legendre immersion and $A$ is a canonical frame field along $f$. Thus, $f$ is an $L$-isothermic immersion (unique up to Laguerre equivalence) and $\Phi$ is its Blaschke potential.

If, for any $m \in \mathbb{R}$, we let

$$
\mathrm{w}_{m}=\mathrm{W}+m e^{-2 u}, \quad \mathrm{~J}_{m}=\mathrm{J}=-\frac{1}{2} e^{-2 u} \Delta u,
$$

then the one-form defined by

$$
\alpha^{(m)}=\left[\begin{array}{cccccc}
0 & 0 & 0 & 0 & 0 & 0 \\
0 & 2 d u & \left(\mathrm{w}_{m}+\mathrm{J}_{m}\right) e^{u} d x & \left(\mathrm{w}_{m}-\mathrm{J}_{m}\right) e^{u} d y & 0 & 0 \\
e^{u} d x & e^{u} d x & 0 & u_{y} d x-u_{x} d y & \left(\mathrm{w}_{m}+\mathrm{J}_{m}\right) e^{u} d x & 0 \\
e^{u} d y & -e^{u} d y & -u_{y} d x+u_{x} d y & 0 & \left(\mathrm{w}_{m}-\mathrm{J}_{m}\right) e^{u} d y & 0 \\
0 & 0 & e^{u} d x & -e^{u} d y & -2 d u & 0 \\
0 & 0 & e^{u} d x & e^{u} d y & 0 & 0
\end{array}\right]
$$

satisfies the Maurer-Cartan integrability condition

$$
d \alpha^{(m)}+\alpha^{(m)} \wedge \alpha^{(m)}=0,
$$

so that there exists a smooth map $A^{(m)}: U \rightarrow L$, such that $d A^{(m)}=A^{(m)} \alpha^{(m)}$. The map $f_{m}: U \rightarrow \boldsymbol{\Lambda}$, given by $f_{m}=\lambda\left(A_{0}^{(m)}, A_{1}^{(m)}\right)$, is a smooth Legendre immersion and $A^{(m)}$ is a canonical frame field along $f_{m}$. Thus, $f_{m}$ is an $L$-isothermic immersion (unique up to Laguerre equivalence) with the same Blaschke potential $\Phi$ as $f=f_{0}$. Then there exists a one-parameter family of non-equivalent $L$-isothermic immersions $\left\{f_{m}\right\}$ with the 
same Blaschke potential $\Phi$. This family amounts to the one-parameter family of Legendre immersions that are $L$-applicable on $f$ (cf. Theorem 4.7).

Actually, any other nondegenerate $L$-isothermic immersion having $\Phi$ as Blaschke potential is Laguerre equivalent to $f_{m}$, for some $m \in \mathbb{R}$.

Definition 4.8. Two $L$-isothermic immersions $f, \tilde{f}$ which are not Laguerre equivalent are said to be T-transforms (spectral deformations) of each other if they have the same Blaschke potential.

REMARK 4.9. The spectral family $f_{m}$ describes all $T$-transforms of $f=f_{0}$. In fact, any nondegenerate $T$-transform of $f$ is Laguerre equivalent to $f_{m}$, for some $m \in \mathbb{R}$. Such a one-parameter family of $L$-isothermic surfaces amounts to the family of second order Laguerre deformations of $f$ in the sense of Remark 4.6

5. $L$-minimal surfaces. Let $f: S \rightarrow \boldsymbol{\Lambda}$ be a Legendre surface. A compactly supported variation of $f$ is a differentiable mapping

$$
V: S \times(-\epsilon, \epsilon) \rightarrow \boldsymbol{\Lambda},(s, t) \longmapsto V(s, t), \quad \text { for some } \epsilon>0,
$$

such that its restriction $f_{t}$ to $S \times\{t\}, t \in(-\epsilon, \epsilon)$, is a Legendre surface, $f_{0}=f$, and such that there exists a compact domain $K \subset S$ for which $V(s, t)=f(s)$, for every $s \in S \backslash K$ and every $t \in(-\epsilon, \epsilon)$. If $f$ is nondegenerate we may suppose that $f_{t}$ is also nondegenerate, for each $t \in(-\epsilon, \epsilon)$. Given a compact domain $C \subset S$, we define the functional

$$
\mathcal{W}_{C}(f)=\int_{C} \Omega_{f}
$$

on the space of smooth Legendre immersions $f: S \rightarrow \boldsymbol{\Lambda}$. We call (5.1) the Weingarten functional. A Legendre immersion $f: S \rightarrow \boldsymbol{\Lambda}$ is called $L$-minimal if it is an extremal of 5.1, that is, if for any compact domain $C \subset S$ and any differentiable variation $f_{t}$ with support in $C$ we have

$$
\left.\frac{d}{d t}\left(\mathcal{W}_{C}\left(f_{t}\right)\right)\right|_{t=0}=0
$$

The following result holds.

TheOREM 5.1 ([43]). A nondegenerate Legendre immersion $f: S \rightarrow \mathbf{\Lambda}$ is L-minimal if and only if $p_{1}+p_{3}=0$.

REMARK 5.2. If $f=(x, n): S \rightarrow \boldsymbol{\Lambda}$ is the Legendre lift of an immersed surface $x: S \rightarrow \mathbb{R}^{3}$, oriented by a unit normal field $n: S \rightarrow S^{2}$, the functional (5.1) coincides with the Weingarten functional

$$
\mathcal{W}(S, x)=\int \frac{H^{2}-K}{K} d A,
$$

where $H$ and $K$ are the mean and Gauss curvatures of $x$ and $d A n$ is the induced area element of the surface. In this case, using the computations made in $\$ 3.4$, one can write the invariant functions $p_{1}$ and $p_{3}$ in terms of the Euclidean invariants and show (cf. e.g. [43]) that the condition to be $L$-minimal is expressed by the nonlinear fourth-order elliptic PDE

$$
\Delta^{\mathrm{III}}\left(\frac{H}{K}\right)=0,
$$


where $\Delta^{\mathrm{III}}$ denotes the Laplace-Beltrami operator with respect to the third fundamental form III of the immersion $x$.

Nondegenerate $L$-minimal surfaces are characterized by the minimality of the Laguerre Gauss map.

Theorem 5.3 ([7], [43]). A nondegenerate Legendre immersion $f: S \rightarrow \boldsymbol{\Lambda}$ is L-minimal if and only if its Laguerre Gauss map $\sigma_{f}: S \rightarrow \mathcal{Q}_{\Sigma} \cong \mathbb{R}^{3,1}$ has zero mean curvature vector.

Proof. According to Remark 3.7, the Laguerre Gauss map $\sigma_{f}: S \rightarrow \mathbb{R}^{3,1}$ is a spacelike immersion with induced metric $\Phi_{f}$ and relative induced area element $\Omega_{f}$. In particular,

$$
\mathcal{W}_{K}(f)=\int_{K} \Omega_{f}=\operatorname{Area}\left(\sigma_{f}\right) \text {. }
$$

From this it is clear that if $\sigma_{f}$ has zero mean curvature vector, then $f$ must be $L$-minimal. For the necessity condition, we need to compute the mean curvature vector of $\sigma_{f}$. The canonical frame field $A$ along $f$ is adapted to the Laguerre Gauss map $\sigma_{f}$. In fact, the bundle induced by $\sigma_{f}$ over $S$ splits into the direct sum

$$
\sigma_{f}^{*}\left(T \mathbb{R}^{3,1}\right)=T\left(\sigma_{f}\right) \oplus N\left(\sigma_{f}\right),
$$

where $T\left(\sigma_{f}\right)=\operatorname{span}\left\{A_{2}, A_{3}\right\}$ is the tangent bundle and $N\left(\sigma_{F}\right)=\operatorname{span}\left\{A_{1}, A_{4}\right\}$ is the normal bundle of $\sigma_{f}$. The metric induced by $\sigma_{f}$ on $S$, is

$$
\Phi_{f}=\left\langle d \sigma_{f}, d \sigma_{f}\right\rangle=\left(\alpha_{0}^{2}\right)^{2}+\left(\alpha_{0}^{3}\right)^{2},
$$

and $\alpha_{0}^{2}, \alpha_{0}^{3}$ defines an orthonormal coframe field on $S$. As $d \sigma_{f}(T S)=\operatorname{span}\left\{A_{2}, A_{3}\right\}$, it follows from (2.7) that

$$
\alpha_{0}^{1}=0=\alpha_{0}^{4} .
$$

Differentiating these equations yields

$$
\begin{aligned}
& 0=d \alpha_{0}^{1}=-\alpha_{2}^{1} \wedge \alpha_{0}^{2}-\alpha_{3}^{1} \wedge \alpha_{0}^{3}, \\
& 0=d \alpha_{0}^{4}=-\alpha_{2}^{4} \wedge \alpha_{0}^{2}-\alpha_{3}^{4} \wedge \alpha_{0}^{3} .
\end{aligned}
$$

Then, by Cartan's Lemma,

$$
\alpha_{i}^{\nu}=h_{i 2}^{\nu} \alpha_{0}^{2}+h_{i 3}^{\nu} \alpha_{0}^{3}, \quad h_{i j}^{\nu}=h_{j i}^{\nu}, \quad \nu=1,4 ; i, j=2,3,
$$

where the functions $h_{i j}^{\nu}$ are the components of the second fundamental form of $\sigma_{f}$,

$$
\Pi=\sum_{i, j=2,3} h_{i j}^{1} \alpha_{0}^{i} \alpha_{0}^{j} \otimes a_{4}+\sum_{i, j=2,3} h_{i j}^{4} \alpha_{0}^{i} \alpha_{0}^{j} \otimes a_{1} .
$$

From (3.14, 3.15) and the symmetry relations 2.8 , it follows that

$$
\left(h_{i j}^{1}\right)=\left[\begin{array}{cc}
1 & 0 \\
0 & -1
\end{array}\right], \quad\left(h_{i j}^{4}\right)=\left[\begin{array}{ll}
p_{1} & p_{2} \\
p_{2} & p_{3}
\end{array}\right] .
$$

The mean curvature vector $\mathbf{H}$ of $\sigma_{f}$ is half the trace of $\Pi$ with respect to $\Phi_{f}$, i.e.,

$$
2 \mathbf{H}=\left(p_{1}+p_{3}\right) A_{1} .
$$

Thus, $\mathbf{H} \equiv 0$ on $S$ if and only if $p_{1}+p_{3}$ vanishes identically on $S$. 
REMARK 5.4. (1) In the presence of umbilical points, one can prove that $\sigma_{f}$ is a conformal harmonic map. (2) The necessity condition in Theorem 5.3 is sort of surprising since there are more variations of the map $\sigma_{f}$ than there are variations through Laguerre Gauss maps. (3) Note that $\mathbf{H}$ is a null section of the normal bundle $N\left(\sigma_{f}\right)$, i.e., $\langle\mathbf{H}, \mathbf{H}\rangle=0$. In particular, it follows from 5.3 that $\sigma_{f}$ is a marginally outer trapped surface (MOTS) in $\mathbb{R}^{3,1}$ (cf. [22, 26] for more details on MOTS and [52] for the analogous situation in Möbius geometry). (4) With respect to the null frame field $\left\{A_{1}, A_{4}\right\}$, the normal connection $\nabla^{\perp}$ in the normal bundle $N(\sigma)$ of $\sigma$ is given by

$$
\nabla^{\perp} a_{1}=\alpha_{1}^{1} \otimes a_{1}, \quad \nabla^{\perp} a_{4}=-\alpha_{1}^{1} \otimes a_{4} .
$$

In particular, we have

$$
2 \nabla^{\perp} \mathbf{H}=\left[d\left(p_{1}+p_{3}\right)+\left(p_{1}+p_{3}\right) \alpha_{1}^{1}\right] a_{1},
$$

so that the parallel condition $\nabla^{\perp} \mathbf{H}=0$ takes the form

$$
d\left(p_{1}+p_{3}\right)+2\left(p_{1}+p_{3}\right)\left(q_{2} \alpha_{0}^{2}-q_{1} \alpha_{0}^{3}\right)=0 .
$$

6. The Pfaffian system of $L$-minimal surfaces. In this section we introduce the Pfaffian differential systems of $L$-minimal surfaces and prove that it is involution. Then, as an application of the general Cartan-Kähler theorem for exterior differential systems in involution we study the Cauchy problem for $L$-minimal surfaces and prove the existence of a unique real analytic $L$-minimal surface passing through a given real analytic integral curve of the system. The functional dependence of the initial curve amounts to the choice of four arbitrary functions in one variable. For the study of the Cauchy problem in other geometric situations we refer to [32, 48, 50,

As shown above, $L$-minimal surfaces are characterized by the condition $p_{1}+p_{3}=0$. Hence, by reasoning as in $\$ 3.3$ they can be interpreted as integral manifolds of the differential system obtained by restricting the Laguerre differential system $(\mathcal{I}, \Omega)$ to the submanifold

$$
Y=\left\{\left(A, q_{1}, q_{2}, p_{1}, p_{2}, p_{3}\right) \in L \times \mathbb{R}^{5}: p_{1}+p_{3}=0\right\} \subset M .
$$

We call $(Y, \mathcal{I}, \Omega)$ the Pfaffian system of L-minimal surfaces. We shall identify $Y$ and $L \times \mathbb{R}^{4}$ and denote by $\left(q_{1}, q_{2}, p_{1}, p_{2}\right)$ the points of $\mathbb{R}^{4}$. In this way, the system $(\mathcal{I}, \Omega)$ on $Y$ is differentially generated by the one-forms

$$
\begin{aligned}
& \eta^{1}=\omega_{0}^{4}, \quad \eta^{2}=\omega_{0}^{1}, \quad \eta^{3}=\omega_{1}^{2}-\omega^{1}, \quad \eta^{4}=\omega_{1}^{3}+\omega^{1}, \\
& \eta^{5}=\omega_{2}^{3}-q_{1} \omega^{1}-q_{2} \omega^{2}, \quad \eta^{6}=\omega_{1}^{1}-2 q_{2} \omega^{1}+2 q_{1} \omega^{2}, \\
& \eta^{7}=\omega_{2}^{1}-p_{1} \omega^{1}-p_{2} \omega^{2}, \quad \eta^{8}=\omega_{3}^{1}-p_{2} \omega^{1}+p_{1} \omega^{2},
\end{aligned}
$$

with independence condition

$$
\omega^{1} \wedge \omega^{2} \neq 0
$$

where $\omega^{1}=\omega_{0}^{2}$ and $\omega^{2}=\omega_{0}^{3}$. Moreover, we let $\pi^{i}=d q_{i}, \zeta^{i}=d p_{i}, i=1,2$, be the set of one-forms that complete $\left\{\eta^{a}, \omega^{i}\right\}$ to a global coframe field on $Y$. 
6.1. Quadratic equations and involution of the system. From the structure equations 2.10 of the Laguerre group, exterior differentiation of $\eta^{1}, \ldots \eta^{8}$ yields

$$
\begin{aligned}
& d \eta^{1} \equiv d \eta^{2} \equiv d \eta^{3} \equiv d \eta^{4} \equiv 0 \bmod \left\{\eta^{a}\right\}, \\
& d \eta^{5} \equiv-\pi^{1} \wedge \omega^{1}-\pi^{2} \wedge \omega^{2}-\left(2 p_{1}+q_{1}^{2}+{q_{2}}^{2}\right) \omega^{1} \wedge \omega^{2} \bmod \left\{\eta^{a}\right\}, \\
& d \eta^{6} \equiv 2 \pi^{1} \wedge \omega^{2}-2 \pi^{2} \wedge \omega^{1}+2 p_{2} \omega^{1} \wedge \omega^{2} \bmod \left\{\eta^{a}\right\}, \\
& d \eta^{7} \equiv-\zeta^{1} \wedge \omega^{1}-\zeta^{2} \wedge \omega^{2}-4\left(q_{1} p_{1}+q_{2} p_{2}\right) \omega^{1} \wedge \omega^{2} \bmod \left\{\eta^{a}\right\}, \\
& d \eta^{8} \equiv \zeta^{1} \wedge \omega^{2}-\zeta^{2} \wedge \omega^{1}-4\left(q_{1} p_{2}-q_{2} p_{1}\right) \omega^{1} \wedge \omega^{2} \bmod \left\{\eta^{a}\right\},
\end{aligned}
$$

where $\left\{\eta^{a}\right\}$ denotes the algebraic ideal generated by the one-forms $\eta^{1}, \ldots \eta^{8}$. The equations in 6.1 are referred to as the quadratic equations of $(Y, \mathcal{I}, \Omega)$. The reduced tableaux matrix $\mu$ of the system is then

$$
{ }^{t} \mu=\left[\begin{array}{cccccccc}
0 & 0 & 0 & 0 & -\pi^{1} & -2 \pi^{2} & -\zeta^{1} & -\zeta^{2} \\
0 & 0 & 0 & 0 & -\pi^{2} & 2 \pi^{1} & -\zeta^{2} & \zeta^{1}
\end{array}\right] .
$$

It then follows that the reduced Cartan characters $s_{1}^{\prime}, s_{2}^{\prime}$ are

$$
s_{1}^{\prime}=4, \quad s_{2}^{\prime}=0 .
$$

Let $V_{2}(\mathcal{I})$ denote the set of all two-dimensional integral elements of $(\mathcal{I}, \Omega)$, i.e.,

$$
V_{2}(\mathcal{I})=\left\{\left(\mathfrak{z}, E_{2}\right) \in G_{2}(T Y): \eta_{\mid E_{2}}^{a}=0, d \eta_{\mid E_{2}}^{a}=0, \omega^{1} \wedge \omega_{\mid E_{2}}^{2} \neq 0\right\},
$$

and let $\mathrm{O}_{2}(\mathcal{I})$ be the open subset of the Grassmannian $G_{2}(T Y)$ consisting of all tangent planes $W \subset T_{\mathfrak{z}} Y$ such that $\omega^{1} \wedge \omega^{2}{ }_{\mid W} \neq 0$. On $O_{2}(\mathcal{I})$, we have fiber coordinates $\left(X_{i}^{a}, V_{i}^{j}, W_{i}^{j}\right), a=1, \ldots, 8, i, j=1,2$, defined by

$$
\eta_{\mid W}^{a}=X_{i}^{a}(W) \omega^{i}{ }_{\mid W}, \quad \pi_{\mid W}^{i}=V_{j}^{i}(W) \omega^{j}{ }_{\mid W}, \quad \zeta_{\mid W}^{i}=W_{j}^{i}(W) \omega^{j}{ }_{\mid W} .
$$

At any point $\mathfrak{z}=\left(A, q_{1}, q_{2}, p_{1}, p_{2}\right)$ of $Y$, the fiber $O_{2}(\mathcal{I})_{\mathfrak{z}}$ is identified with the affine space $\mathbb{A}^{24}$ by the map

$$
\mathrm{O}_{2}(\mathcal{I})_{\mathfrak{z}} \ni W \longmapsto\left(X_{i}^{a}(W), V_{i}^{j}(W), W_{i}^{j}(W)\right) .
$$

From the quadratic equations 6.1 it follows that $V_{2}(\mathcal{I})_{\mathfrak{z}}$ is the 4-dimensional affine subspace of $\mathrm{O}_{2}(\mathcal{I})_{\mathfrak{z}}$ defined by the equations

$$
\begin{aligned}
& X_{i}^{a}=0, \quad a=1, \ldots, 8 ; i=1,2 \\
& V_{2}^{1}-V_{1}^{2}=2 p_{1}+q_{1}^{2}+q_{2}^{2}, \\
& V_{1}^{1}+V_{2}^{2}=-p_{2}, \\
& W_{2}^{1}-W_{1}^{2}=4\left(q_{1} p_{1}+q_{2} p_{2}\right), \\
& W_{1}^{1}+W_{2}^{2}=4\left(q_{1} p_{2}-q_{2} p_{1}\right) .
\end{aligned}
$$

This yields the following.

LEMma 6.1. The set $V_{2}(\mathcal{I})$ of two-dimensional integral elements of the Pfaffian system $(Y, \mathcal{I}, \Omega)$ is a real analytic 18-dimensional embedded submanifold of the Grassmannian $G_{2}(T Y)$ and the fiber over $\mathfrak{z} \in Y$ of the bundle map $V_{2}(\mathcal{I}) \rightarrow Y,\left(\mathfrak{z}, E_{2}\right) \mapsto \mathfrak{z}$, is a 4-dimensional affine subspace of $G_{2}\left(T_{\mathfrak{z}} Y\right)$, for each $\mathfrak{z} \in Y$, i.e.,

$$
t=\operatorname{dim} V_{2}(\mathcal{I})_{\mathfrak{z}}=4, \quad \text { for each } \mathfrak{z} \in Y \text {. }
$$


From this and $(6.2)$, it follows that $t=s_{1}^{\prime}+2 s_{2}^{\prime}$, so that Cartan's test of involution applies. We can then state the following.

Proposition 6.2. The Pfaffian differential system $(Y, \mathcal{I}, \Omega)$ of L-minimal surfaces is in involution ${ }^{6}$ and its integral manifolds depend on four functions in one variable.

6.2. Polar equations and the Cauchy problem. Next, we study the polar equations of the system $(Y, \mathcal{I}, \Omega)$. On the tangent bundle $T Y$, we consider fiber coordinates $\left(a^{i}, X^{a}, V^{i}, W^{i}\right)$ defined, for any tangent vector $\xi$, by

$$
a^{i}(\xi)=\omega^{i}(\xi), \quad X^{a}(\xi)=\eta^{a}(\xi), \quad V^{i}(\xi)=\pi^{i}(\xi), \quad W^{i}(\xi)=\zeta^{i}(\xi) .
$$

Thus, $\left(a^{i}, X^{a}, V^{i}, W^{i}\right)$ can be used as homogeneous fiber coordinates on the Grassmannian $G_{1}(T Y)$.

A one-dimensional integral element of $(\mathcal{I}, \Omega)$, at a fixed point $\mathfrak{z} \in Y$, is a onedimensional subspace $E_{1}=\operatorname{span}\{\xi\}$ of $T_{\mathfrak{z}} Y$, spanned by a nonzero tangent vector $\xi$, such that

$$
\eta^{a}(\xi)=0, a=1, \ldots, 8 ; \quad \omega^{1}(\xi) \omega^{1}(\xi)+\omega^{2}(\xi) \omega^{2}(\xi) \neq 0 .
$$

Let $V_{1}(\mathcal{I})$ denote the set of all one-dimensional integral elements of $(\mathcal{I}, \Omega)$. The set $V_{1}(\mathcal{I})$ is the submanifold of $G_{1}(T Y)$ defined by the equations

$$
X^{a}=0, \quad a=1, \ldots, 8 ; \quad\left(a^{1}\right)^{2}+\left(a^{2}\right)^{2} \neq 0 .
$$

Let $\left(\mathfrak{z}, E_{1}\right)$ be a one-dimensional integral element. We recall that the polar or extension space $H\left(\mathfrak{z}, E_{1}\right)$ of $\left(\mathfrak{z}, E_{1}\right)$ is the subspace of $T_{\mathfrak{z}} Y$ defined by the polar equations

$$
\eta^{a}=0, \quad \imath_{\xi} d \eta^{a}=0, \quad a=1, \ldots, 8 .
$$

Let $\mathfrak{z}=\left(A, q_{1}, q_{2}, p_{1}, p_{2}\right) \in Y$ and $\left(\mathfrak{z}, E_{1}\right)$ a one-dimensional integral element with $E_{1}=\operatorname{span}\{\xi\}, \xi \neq 0$. From 6.8, 6.7), 6.5, using the quadratic equations 6.1 of the system, the polar equations read

$$
\begin{aligned}
& \eta^{a}=0, \quad a= 1, \ldots, 8, \\
& a^{1} \pi^{1}+a^{2} \pi^{2}+ {\left[a^{2}\left(2 p_{1}+q_{1}^{2}+q_{2}^{2}\right)-V^{1}\right] \omega^{1} } \\
&- {\left[a^{1}\left(2 p_{1}+q_{1}^{2}+q_{2}^{2}\right)+V^{2}\right] \omega^{2}=0, } \\
&-a^{2} \pi^{1}+a^{1} \pi^{2}-\left[V^{2}+p_{2} a^{2}\right] \omega^{1}+\left[V^{1}+p_{2} a^{1}\right] \omega^{2}=0, \\
& a^{1} \zeta^{1}+a^{2} \zeta^{2}-\left[W^{1}+4 a^{1}\left(q_{1} p_{1}+q_{2} p_{2}\right)\right] \omega^{1} \\
&-\left[W^{2}-4 a^{2}\left(q_{1} p_{1}+q_{2} p_{2}\right)\right] \omega^{2}=0, \\
&-a^{2} \zeta^{1}+a^{1} \zeta^{2}-\left[W^{2}-4 a^{2}\left(q_{1} p_{2}-q_{2} p_{1}\right)\right] \omega^{1} \\
&+\left[W^{1}-4 a^{1}\left(q_{1} p_{2}-q_{2} p_{1}\right)\right] \omega^{2}=0 .
\end{aligned}
$$

The polar equations have maximal rank and then the polar space $H\left(\mathfrak{z}, E_{1}\right)$ has dimension two, for every $\left(\mathfrak{z}, E_{1}\right) \in V_{1}(\mathcal{I})$. Therefore, we are led to the following.

\footnotetext{
${ }^{6}$ that is, at every point $\mathfrak{z} \in Y$ there exists an ordinary integral element $\left(\mathfrak{z}, E_{2}\right) \in V_{2}(\mathcal{I})_{\mathfrak{z}}(\mathrm{cf}$. 15. for more details).
} 
Lemma 6.3. For any $\left(\mathfrak{z}, E_{1}\right) \in V_{1}(\mathcal{I})$, the polar space $H\left(\mathfrak{z}, E_{1}\right)$ is the unique two-dimensional integral element of $(\mathcal{I}, \Omega)$ such that $E_{1} \subset H\left(\mathfrak{z}, E_{1}\right)$. According to 6.7$)$, the set $V_{1}(\mathcal{I})$ is a 19-dimensional embedded submanifold of $G_{1}(T Y)$, and hence any $\left(\mathfrak{z}, E_{1}\right) \in V_{1}(\mathcal{I})$ is Kähler-regular and

$$
(0)_{\mathfrak{z}} \subset\left(\mathfrak{z}, E_{1}\right) \subset H\left(\mathfrak{z}, E_{1}\right)
$$

is a regular flag (cf. [25, p. 42]).

By the Cartan-Kähler theorem (cf. [25, p. 42], or [15, p. 81]) we have the following. Lemma 6.4. Let $\gamma:(-\epsilon, \epsilon) \rightarrow Y$ be a real analytic embedded curve such that $\operatorname{span}\{\dot{\gamma}(t)\} \in$ $V_{1}(\mathcal{I})$, for each $t \in(-\epsilon, \epsilon)$. Then there exists a unique real analytic connected integral manifold $\Sigma \subset Y$ of $(\mathcal{I}, \Omega)$ such that $\gamma \subset \Sigma$. The manifold $\Sigma$ is unique in the sense that any other integral manifold of $(Y, \mathcal{I}, \Omega)$ with these properties agrees with $\Sigma$ on an open neighborhood of $\gamma$.

This has the following geometric consequence.

TheOrem 6.5 (The Cauchy problem). Let $s_{1}, s_{2}, r_{1}, r_{2}:(-\epsilon, \epsilon) \rightarrow \mathbb{R}$ be real analytic functions defined on an open interval, and let $B \in L$ be an element of the Laguerre group. There exists an open neighborhood $U \subset \mathbb{R}^{2}$ of the origin and a unique real analytic $L$-minimal framed surface $(U, f, A)$, satisfying the following initial conditions

1. $A(0,0)=B, q_{i}(t, 0)=s_{i}(t), p_{i}(t, 0)=r_{i}(t)$, for each $t \in(-\epsilon, \epsilon) \cap U$,

2. $\alpha_{0 \mid(-\epsilon, \epsilon) \cap U}^{2}=A^{*}\left(\omega_{0}^{2}\right)_{\mid(-\epsilon, \epsilon) \cap U}=d t, \alpha_{0 \mid(-\epsilon, \epsilon) \cap U}^{3}=A^{*}\left(\omega_{0}^{3}\right)_{\mid(-\epsilon, \epsilon) \cap U}=0$,

where $q_{i}, p_{i}, i=1,2$, are the invariant functions of $f$.

Proof. Given the real analytic functions $s_{i}$ and $r_{i}$, consider the l-valued one-form $\beta$ defined on $(-\epsilon, \epsilon)$ given by

$$
\beta=\left[\begin{array}{cccccc}
0 & 0 & 0 & 0 & 0 & 0 \\
0 & 2 s_{2} & r_{1} & r_{2} & 0 & 0 \\
1 & 1 & 0 & -s_{1} & r_{1} & 0 \\
0 & 0 & s_{1} & 0 & r_{2} & 0 \\
0 & 0 & 1 & 0 & -2 s_{2} & 0 \\
0 & 0 & 1 & 0 & 0 & 0
\end{array}\right] d t .
$$

Let $\Gamma:(-\epsilon, \epsilon) \rightarrow L$ be the unique real analytic map such that

$$
\Gamma^{*}(\omega)=\beta, \quad \Gamma(0)=B .
$$

We may suppose that $\Gamma$ is an embedding, by possibly choosing a smaller $\epsilon$. Next, let $\gamma:(-\epsilon, \epsilon) \rightarrow Y$ be the curve defined by $\gamma(t):=\left(\Gamma(t), s_{1}(t), s_{2}(t), r_{1}(t), r_{2}(t)\right)$, for each $t$. By construction, $\gamma$ is a real analytic embedded curve such that $\operatorname{span}\{\dot{\gamma}(t)\} \in V_{1}(\mathcal{I})$, for each $t \in(-\epsilon, \epsilon)$. According to Lemma 6.4 there exists a unique real analytic embedded solution of the system $(Y, \mathcal{I}, \Omega)$, say $\Sigma=\left(A, q_{1}, q_{2}, p_{1}, p_{2}\right): U \rightarrow Y$, defined on an open disk $U$ containing $(-\epsilon, \epsilon) \times\{0\}$, such that $\Sigma(t, 0)=\gamma(t)$, for each $t \in(-\epsilon, \epsilon)$. The framed immersion $(U, f, A)$, where $f=\pi_{L} \circ A$, is $L$-minimal and $q_{1}, q_{2}, p_{1}, p_{2}$ are the invariant functions of $f$. Thus $(U, f, A)$ satisfies the required conditions. 
REMARK 6.6. Note that the functional dependence of the general solutions of the system $(Y, \mathcal{I}, \Omega)$ agrees with that of the initial data of Theorem 6.5. Specifying the initial data of Theorem 6.5 amounts to the choice of four arbitrary functions in one variable.

7. Generalized $L$-minimal surfaces. Let $S$ be an oriented surface and let $f: S \rightarrow \boldsymbol{\Lambda}$ be a nondegenerate Legendre immersion into the Laguerre space. Let $A: S \rightarrow L$ be a canonical frame field along $f$. The Laguerre metric $\left(\alpha_{0}^{2}\right)^{2}+\left(\alpha_{0}^{3}\right)^{2}$ and the area element $\alpha_{0}^{2} \wedge \alpha_{0}^{3}$ induced by $A$ determine on $S$ an oriented conformal structure and hence, by the existence of isothermal coordinates, a unique compatible complex structure which makes $S$ into a Riemann surface. In terms of the canonical frame field $A$, the complex structure is characterized by the property that the complex-valued one-form

$$
\varphi=\alpha_{0}^{2}+i \alpha_{0}^{3}
$$

is of type $(1,0)$. Taking into account Remark 3.12 , the complex-valued quartic differential form given by

$$
\mathfrak{Q}_{f}=Q \varphi^{4}, \quad Q:=\frac{1}{2}\left(p_{1}-p_{3}\right)-i p_{2},
$$

and the complex-valued quadratic differential form given by

$$
\mathfrak{P}_{f}=P \varphi^{2}, \quad P:=p_{1}+p_{3}
$$

are globally defined on the Riemann surface $S$.

REMARK 7.1. The quartic differential $\mathfrak{Q}_{f}$ was considered by the authors in [43], where it was proved that for $L$-minimal surfaces $\mathfrak{Q}_{f}$ is holomorphic. Notice that the quadratic differential $\mathfrak{P}_{F}$ vanishes exactly for $L$-minimal surfaces.

Definition 7.2. A nondegenerate Legendre immersion $f: S \rightarrow \boldsymbol{\Lambda}$ is called a generalized $L$-minimal surface if the quartic differential $\mathfrak{Q}_{f}$ is holomorphic $]^{7}$

REMARK 7.3 (Surfaces in Möbius geometry). The results discussed in this section can be considered as the Laguerre geometric counterpart of well-known results for surfaces in Möbius geometry (cf. [27, 33 for more details). For the sake of completeness, we briefly recall some of them. Many features of CMC surfaces in three-dimensional space forms, viewed as isothermic surfaces in Möbius space $S^{3}$, can be interpreted in terms of the transformation theory of isothermic surfaces. More specifically, it can be proved that CMC surfaces in space forms arise in associated one-parameter families as $T$-transforms of minimal surfaces in space forms [4, 17, 18, 27, 33. Minimal surfaces in space forms are isothermic and Willmore, that is, are critical points of the Willmore energy $\int\left(H^{2}-K\right) d A$ (cf. [7, 13, 64]). By a classical result of Thomsen [27, 33, 64], a Willmore surface without umbilics is isothermic if and only if it is locally Möbius equivalent to a minimal surface in some space form. K. Voss obtained a uniform Möbius geometric characterization of Willmore surfaces and CMC surfaces in space forms using the differential $(4,0)$ form $\mathfrak{Q}$

\footnotetext{
${ }^{7}$ The name is motivated by the name "generalized Willmore surfaces" ("verallgemeinerte Willmore-Flächen") used by K. Voss [66 to indicate those surfaces in conformal geometry with holomorphic Bryant differential $(4,0)$ form $[13$. The name "generalized Laguerre minimal surfaces" was also used with a different meaning in 2] to indicate those $L$-minimal surfaces for which the Gauss curvature $K$ is allowed to vanish on a set of isolated points.
} 
introduced by Bryant [13] for Willmore surfaces (cf. also [12, 11, 33, 66]). Voss observed that $\mathfrak{Q}$, which indeed may be defined for any conformal immersion of a Riemann surface $M$ into $S^{3}$, is holomorphic if and only if, locally and away from umbilics and isolated points, the immersion is Willmore or has constant mean curvature in some space form embedded in $S^{3}$.

It is quite easy to prove the following facts. For the proof we refer to [53].

Lemma 7.4. Let $f: S \rightarrow \boldsymbol{\Lambda}$ be a nondegenerate Legendre immersion. Then:

1. The quartic differential $\mathfrak{Q}_{f}$ is holomorphic if and only if

$$
d Q \wedge \varphi=-4\left(q_{2} \alpha_{0}^{2}-q_{1} \alpha_{0}^{3}\right) Q \wedge \varphi .
$$

2. $\mathfrak{Q}_{f}$ is holomorphic if and only if the Laguerre Gauss map of $f$ has parallel mean curvature vector.

3. If $\mathfrak{Q}_{f}$ is holomorphic, then $\mathfrak{P}_{f}$ is holomorphic.

4. If $\mathfrak{Q}_{f}$ is holomorphic and $\mathfrak{P}_{f}$ is non-zero, then $f$ is L-isothermic.

5. If $\mathfrak{Q}_{f}$ is holomorphic and $\mathfrak{P}_{f} \neq 0$, then $\mathfrak{Q}_{f}=c \mathfrak{P}_{f}^{2}$, for $c \in \mathbb{R}$.

We are now ready to prove our next result.

Proposition 7.5. A nondegenerate Legendre immersion $f: S \rightarrow \Lambda$ is generalized L-minimal if and only if it is L-minimal, in which case the quadratic differential $\mathfrak{P}_{F}$ vanishes on $S$, or it is L-isothermic with Blaschke potential $\Phi=e^{u}$ satisfying the second order partial differential equation

$$
\Delta u=c e^{-2 u}
$$

where $c$ is a real constant.

Proof. If $\mathfrak{Q}_{f}$ is holomorphic and the holomorphic quartic differential $\mathfrak{P}_{f}$ vanishes, then $f$ is $L$-minimal. If instead $\mathfrak{P}_{f}$ is nowhere vanishing, then $f$ is $L$-isothermic by Lemma 7.4. Let $z=x+i y: U \subset S \rightarrow \mathbb{C}$ be an isothermic chart, so that the canonical coframing $\left(\alpha_{0}^{2}, \alpha_{0}^{3}\right)$ takes the form $\alpha_{0}^{2}=e^{u} d x$ and $\alpha_{0}^{3}=e^{u} d y$, where $\Phi=e^{u}$ is the Blaschke potential (cf. Section 4).

From 4.1 and 4.2 we get

$$
\mathfrak{Q}_{f}=\frac{1}{2}\left(p_{1}-p_{3}\right) \omega^{4}=\mathrm{J} e^{4 u}(d z)^{4}=-\frac{1}{2}\left(e^{-2 u} \Delta u\right) e^{4 u}(d z)^{4} .
$$

Since $\mathfrak{Q}_{f}$ is holomorphic,

$$
\left(e^{-2 u} \Delta u\right) e^{4 u}=c,
$$

for a constant $c \in \mathbb{R}$, that is

$$
\Delta u=c e^{-2 u} .
$$

Conversely, if we assume that $\Phi=e^{u}$ satisfy the equation $(7.5)$, then the right hand side of (4.3) vanishes identically, which implies that $p_{1}+p_{3}=k e^{-2 u}$, for a constant $k \in \mathbb{R}$. A direct computation shows that $p_{1}+p_{3}=k e^{-2 u}$ satisfies the equation

$$
d\left(p_{1}+p_{3}\right)+2\left(p_{1}+p_{3}\right)\left(q_{2} \alpha_{0}^{2}-q_{1} \alpha_{0}^{3}\right)=0 .
$$

This expresses the fact that the $L$-Gauss map of $f, \sigma_{f}=\left[A_{0}\right]$, has parallel mean curvature vector, or equivalently, that the quartic differential $\mathfrak{Q}_{f}$ is holomorphic. 


\subsection{Special $L$-isothermic surfaces and $T$-transforms}

Definition 7.6. A nondegenerate $L$-isothermic immersion $f: S \rightarrow \boldsymbol{\Lambda}$ is called special if its Blaschke potential $\Phi=e^{u}$ satisfies the second order PDE (7.5), i.e.,

$$
\Delta u=c e^{-2 u}, \quad c \in \mathbb{R} .
$$

The constant $c$ is called the character of the special $L$-isothermic surface $f$.

EXAMPLE 7.7 ( $L$-minimal isothermic surfaces). Since $L$-minimal surfaces are characterized by the condition $p_{1}+p_{3}=0$, if a nondegenerate $L$-isothermic immersion $f: S \rightarrow \boldsymbol{\Lambda}$ is also $L$-minimal, i.e., $p_{2}=0$, then the right hand side of 4.3 is identically zero. This implies that $d\left(e^{2 u} \Delta u\right)=0$, and hence the following.

Proposition 7.8. Any nondegenerate L-minimal isothermic immersion $f: S \rightarrow \boldsymbol{\Lambda}$ is special L-isothermic.

Other examples of $L$-minimal isothermic surfaces include $L$-minimal canal surfaces [42, 46.

Let $f: S \rightarrow \boldsymbol{\Lambda}$ be a special $L$-isothermic immersion. From the proof of Proposition 7.5, the invariants $\mathrm{J}$ and $\mathrm{W}$ of $f$ are given by

$$
\mathrm{W}=k e^{-2 u}, \quad \mathrm{~J}=-\frac{1}{2} e^{-2 u} \Delta u,
$$

where $k$ is a real constant.

Definition 7.9. The constant $k$ will be referred to as the deformation (or spectral) parameter of the special $L$-isothermic immersion $F$.

We have the following.

Proposition 7.10. Any special L-isothermic immersion is the T-transform of an L-minimal isothermic immersion.

Proof. According to Section 44 there exists, up to Laguerre equivalence, a unique $L$-isothermic immersion $f$ with Blaschke potential $\Phi=e^{u}$ satisfying $(7.5)$ and with invariant functions

$$
\mathrm{W}=0, \quad \mathrm{~J}=-\frac{1}{2} e^{-2 u} \Delta u=-\frac{c}{2} e^{-4 u} .
$$

Since $\mathrm{w}=0, f$ is $L$-minimal. Next, let $\tilde{f}$ be a special $L$-isothermic immersion with the same Blaschke potential $\Phi$ as $f$ and with deformation parameter $k$. The discussion in Section 4 implies that $\tilde{f}$ is a $T_{m}$-transform of $f$. The invariants of $\tilde{f}$ are then given by

$$
\mathrm{W}_{m}=m e^{-2 u}, \quad \mathrm{~J}_{m}=\mathrm{J}=-\frac{c}{2} e^{-4 u} .
$$

From (7.6) and (7.7), it follows that $m=k$.

From Propositions 7.5 7.8, and Proposition 7.10, we have the following.

THEOREM 7.11 ([53]). A nondegenerate Legendre immersion $f: M \rightarrow \Lambda$ is generalized $L$-minimal if and only if the immersion $f$ is L-minimal, in which case $\mathfrak{P}_{f}$ vanishes, or is locally the T-transform of an L-minimal isothermic surface.

In particular, if $f$ has holomorphic $\mathfrak{Q}_{f}$ and zero $\mathfrak{P}_{f}$, then $f$ is $L$-isothermic if and only if it is $L$-minimal isothermic. 
$L$-minimal isothermic surfaces and their $T$-transforms (i.e., special $L$-isothermic surfaces with non-zero deformation parameter) can be characterized in terms of the differential geometry of their Laguerre Gauss maps. The following result was proved in [53].

TheOREM 7.12 ([53]). Let $f: S \rightarrow \boldsymbol{\Lambda}$ be a nondegenerate Legendre immersion. Then:

1. $f$ is L-minimal and L-isothermic if and only if its Laguerre Gauss map $\sigma_{f}: S \rightarrow$ $\mathbb{R}^{3,1} \cong \mathcal{Q}_{\Sigma}$ has zero mean curvature in some spacelike, timelike, or (degenerate) isotropic hyperplane of $\mathbb{R}^{3,1}$.

2. $f$ is generalized L-minimal and non-zero $\mathfrak{P}_{F}$ if and only if its Laguerre Gauss map $\sigma_{f}: S \rightarrow \mathbb{R}^{3,1}$ has constant mean curvature $H=r$ in some translate of hyperbolic three-space $\mathbb{H}^{3}\left(-r^{2}\right) \subset \mathbb{R}^{3,1}$, de Sitter three-space $\mathbb{S}_{1}^{3}\left(r^{2}\right) \subset \mathbb{R}^{3,1}$, or has zero mean curvature in some translate of a time-oriented lightcone $\mathcal{L}_{ \pm}^{3} \subset \mathbb{R}^{3,1}$.

In addition, if the Laguerre Gauss map of $f$ takes values in a spacelike (respectively, timelike, isotropic) hyperplane, then the Laguerre Gauss maps of the T-transforms of $f$ take values in a translate of a hyperbolic three-space (respectively, de Sitter three-space, time-oriented lightcone). In particular, the signature of the three-space where the Laguerre Gauss map takes values remains unchanged under T-transformations.

REMARK 7.13. As an application of these results, one can show (cf. [53]) that the Lawson correspondence [35] between certain isometric CMC surfaces in different hyperbolic three-spaces and, in particular, the Umehara-Yamada isometric perturbation [65] of minimal surfaces of $\mathbb{R}^{3}$ into CMC surfaces in hyperbolic three-space, can be viewed as a special case of the $T$-transformation of $L$-isothermic surfaces with holomorphic quartic differential. (For a Möbius geometric interpretation of the Umehara-Yamada perturbation see [28, 51]). This interpretation also applies to the generalizations of Lawson's correspondence in the Lorentzian [54 and the (degenerate) isotropic situations, namely to the perturbation of maximal surfaces in Minkowski three-space into CMC spacelike surfaces in de Sitter three-space [1, 3, 34, 36], and that of zero mean curvature spacelike surfaces in a (degenerate) isotropic three-space into zero mean curvature spacelike surfaces in a time-oriented lightcone of $\mathbb{R}^{3,1}$.

Acknowledgments. Authors partially supported by MIUR (Italy) under the PRIN project Varietà reali e complesse: geometria, topologia e analisi armonica; and by the GNSAGA of INDAM. The present research was also partially supported by MIUR grant "Dipartimenti di Eccellenza" 2018-2022, CUP: E11G18000350001, DISMA, Politecnico di Torino.

\section{References}

[1] R. Aiyama, K. Akutagawa, Kenmotsu-Bryant type representation formulas for constant mean curvature surfaces in $H^{3}\left(-c^{2}\right)$ and $S_{1}^{3}\left(c^{2}\right)$, Ann. Global Anal. Geom. 17 (1999), 49-75.

[2] J. A. Aledo, J. A. Gálvez, V. Lozano, Complete Laguerre minimal surfaces in $\mathbb{R}^{3}$, Nonlinear Anal. 92 (2013), 1-12. 
[3] J. A. Aledo, J. A. Gálvez, P. Mira, Marginally trapped surfaces in $\mathbb{L}^{4}$ and an extended Weierstrass-Bryant representation, Ann. Global Anal. Geom. 28 (2005), 395-415.

[4] L. Bianchi, Complementi alle ricerche sulle superficie isoterme, Ann. Mat. Pura Appl. 12 (1905), 19-54.

[5] L. Bianchi, Lezioni di geometria diferenziale, terza edizione, Zanichelli, Bologna, 1927.

[6] W. Blaschke, Über die Geometrie von Laguerre: I, Abh. Math. Sem. Univ. Hamburg 3 (1924), 176-194 II, Abh. Math. Sem. Univ. Hamburg 3 (1924), 195-212 III, Abh. Math. Sem. Univ. Hamburg 4 (1925), 1-12.

[7] W. Blaschke, Vorlesungen über Differentialgeometrie. III: Differentialgeometrie der Kreise und Kugeln, bearbeitet von G. Thomsen, Grundlehren Math. Wiss. 29, Springer, Berlin, 1929.

[8] A. I. Bobenko, T. Hoffmann, B. A. Springborn, Minimal surfaces from circle patterns: geometry from combinatorics, Ann. of Math. (2) 164 (2006), 231-264.

[9] A. I. Bobenko, H. Pottmann, J. Wallner, A curvature theory for discrete surfaces based on mesh parallelity, Math. Ann. 348 (2010), 1-24.

[10] A. I. Bobenko, Y. Suris, On organizing principles of discrete differential geometry. Geometry of spheres, Uspekhi Mat. Nauk 62 (2007), no. 1, 3-50; English transl.: Russian Math. Surveys 62 (2007), no. 1, 1-43.

[11] C. Bohle, Constant mean curvature tori as stationary solutions to the Davey-Stewartson equation, Math. Z. 271 (2012), 489-498.

[12] C. Bohle, G. P. Peters, Bryant surfaces with smooth ends, Comm. Anal. Geom. 17 (2009), 587-619.

[13] R. L. Bryant, A duality theorem for Willmore surfaces, J. Differential Geom. 20 (1984), $23-53$.

[14] R. L. Bryant, Surfaces of mean curvature one in hyperbolic space, in: Théorie des variétés minimales et applications (Palaiseau, 1983-1984), Astérisque 154-155 (1987), 321-347.

[15] R. L. Bryant, S.-S. Chern, R. B. Gardner, H. L. Goldschmidt, P. A. Griffiths, Exterior Differential Systems, Math. Sci. Res. Inst. Publ. 18, Springer, New York, 1991.

[16] R. L. Bryant, S.-S. Chern, P. A. Griffiths, Exterior differential systems, in: Proceedings of the 1980 Beijing Symposium on Differential Geometry and Differential Equations, Vol. 1, Sci. Press Beijing, Beijing, 1982, 219-338.

[17] P. Calapso, Sulla superficie a linee di curvatura isoterme, Rend. Circ. Mat. Palermo 17 (1903), 275-286.

[18] P. Calapso, Sulle trasformazioni delle superficie isoterme, Ann. Mat. Pura Appl. 24 (1915), 11-48.

[19] E. Cartan, Sur le problème général de la déformation, C. R. Congrés Strasbourg (1920), 397-406; or Oeuvres Complètes, III 1, 539-548.

[20] É. Cartan, Les systèmes différentiels extérieurs et leurs applications géométriques, Actualités Sci. Ind., no. 994, Hermann et Cie., Paris, 1945.

[21] T. E. Cecil, Lie Sphere Geometry. With Applications to Submanifolds, second ed., Universitext, Springer, New York, 2008.

[22] P. T. Chruściel, G. J. Galloway, D. Pollack, Mathematical general relativity: a sampler, Bull. Amer. Math. Soc. (N.S.) 47 (2010), 567-638.

[23] P. A. Griffiths, On Cartan's method of Lie groups and moving frames as applied to uniqueness and existence questions in differential geometry, Duke Math. J. 41 (1974), 775-814.

[24] P. A. Griffiths, Exterior Differential Systems and the Calculus of Variations, Progr. Math. 25, Birkhäuser, Boston, 1982. 
[25] P. A. Griffiths, G. R. Jensen, Differential Systems and Isometric Embeddings, Ann. of Math. Stud. 114, Princeton Univ. Press, Princeton, NJ, 1987.

[26] S. W. Hawking, G. F. R. Ellis, The Large Scale Structure of Space-time, Cambridge Monographs on Mathematical Physics 1, Cambridge Univ. Press, London, 1973.

[27] U. Hertrich-Jeromin, Introduction to Möbius Differential Geometry, London Math. Soc. Lecture Note Ser. 300, Cambridge Univ. Press, Cambridge, 2003.

[28] U. Hertrich-Jeromin, E. Musso, L. Nicolodi, Möbius geometry of surfaces of constant mean curvature 1 in hyperbolic space, Ann. Global Anal. Geom. 19 (2001), 185-205.

[29] T. A. Ivey, J. M. Landsberg, Cartan for Beginners: Differential Geometry via Moving Frames and Exterior Differential Systems, Grad. Stud. Math. 61, Amer. Math. Soc., Providence, RI, 2003.

[30] G. R. Jensen, Deformation of submanifolds of homogeneous spaces, J. Differential Geom. 16 (1981), 213-246.

[31] G. R. Jensen, Lie sphere geometry, this volume, 211-222.

[32] G. R. Jensen, E. Musso, L. Nicolodi, The geometric Cauchy problem for the membrane shape equation, J. Phys. A 47 (2014), no. 49, 495201.

[33] G. R. Jensen, E. Musso, L. Nicolodi, Surfaces in Classical Geometries. A Treatment by Moving Frames, Universitext, Springer, Cham, 2016.

[34] O. Kobayashi, Maximal surfaces in the 3-dimensional Minkowski space $L^{3}$, Tokyo J. Math. 6 (1983), 297-309.

[35] H. B. Lawson, Complete minimal surfaces in $S^{3}$, Ann. of Math. (2) 92 (1970), 335-374.

[36] S. Lee, Spacelike surfaces of constant mean curvature \pm 1 in de Sitter 3 -space $\mathbb{S}_{1}^{3}(1)$, Illinois J. Math. 49 (2005), 63-98.

[37] T. Li, H. Li, C. Wang, Classification of hypersurfaces with parallel Laguerre second fundamental form in $\mathbb{R}^{n}$, Differential Geom. Appl. 28 (2010), 148-157.

[38] T. Li, C. P. Wang, Laguerre geometry of hypersurfaces in $\mathbb{R}^{n}$, Manuscripta Math. 122 (2007), 73-95.

[39] J. M. Manzano, E. Musso, L. Nicolodi, Björling type problems for elastic surfaces, Rend. Semin. Mat. Univ. Politec. Torino 74 (2016), 213-233.

[40] B. McKay, Introduction to exterior differential systems, this volume, 45-55.

[41] E. Musso, Deformation of surfaces in Möbius space, Rend. Istit. Mat. Univ. Trieste 27 (1995), 25-45.

[42] E. Musso, L. Nicolodi, L-minimal canal surfaces, Rend. Mat. Appl. (7) 15 (1995), 421-445.

[43] E. Musso, L. Nicolodi, A variational problem for surfaces in Laguerre geometry, Trans. Amer. Math. Soc. 348 (1996), 4321-4337.

[44] E. Musso, L. Nicolodi, Isothermal surfaces in Laguerre geometry, Boll. Un. Mat. Ital. B (7) 11 (1997), no. 2, suppl., 125-144.

[45] E. Musso, L. Nicolodi, On the equation defining isothermic surfaces in Laguerre geometry, in: New Developments in Differential Geometry, Budapest 1996, Kluwer Acad. Publ., Dordrecht, 1999, 285-294.

[46] E. Musso, L. Nicolodi, Laguerre geometry of surfaces with plane lines of curvature, Abh. Math. Sem. Univ. Hamburg 69 (1999), 123-138.

[47] E. Musso, L. Nicolodi, The Bianchi-Darboux transform of L-isothermic surfaces, Internat. J. Math. 11 (2000), 911-924.

[48] E. Musso, L. Nicolodi, On the Cauchy problem for the integrable system of Lie minimal surfaces, J. Math. Phys. 46 (2005), 113509. 
[49] E. Musso, L. Nicolodi, Deformation and applicability of surfaces in Lie sphere geometry, Tohoku Math. J. (2) 58 (2006), 161-187.

[50] E. Musso, L. Nicolodi, A class of overdetermined systems defined by tableaux: involutiveness and the Cauchy problem, Phys. D 229 (2007), 35-42.

[51] E. Musso, L. Nicolodi, Conformal deformation of spacelike surfaces in Minkowski space, Houston J. Math. 35 (2009), 1029-1049.

[52] E. Musso, L. Nicolodi, Marginally outer trapped surfaces in de Sitter space by low-dimensional geometries, J. Geom. Phys. 96 (2015), 168-186.

[53] E. Musso, L. Nicolodi, Holomorphic differentials and Laguerre deformation of surfaces, Math Z. 284 (2016), 1089-1110.

[54] B. Palmer, Spacelike constant mean curvature surfaces in pseudo-Riemannian space forms, Ann. Global Anal. Geom. 8 (1990), 217-226.

[55] B. Palmer, Remarks on a variational problem in Laguerre geometry, Rend. Mat. Appl. (7) 19 (1999), 281-293.

[56] B. Palmer, Anisotropic wavefronts and Laguerre geometry, J. Math. Phys. 56 (2015), no. 2, 023503.

[57] M. Pember, Lie applicable surfaces, Comm. Anal. Geom., to appear, arXiv:1606.07205 $[$ math.DG]

[58] H. Pottmann, P. Grohs, N. J. Mitra, Laguerre minimal surfaces, isotropic geometry and linear elasticity, Adv. Comput. Math. 31 (2009), 391-419.

[59] H. Pottmann, M. Peternell, Applications of Laguerre geometry in CAGD, Comput. Aided Geom. Design 15 (1998), 165-186.

[60] C. Rogers, A. Szereszewski, A Bäcklund transformation for L-isothermic surfaces, J. Phys. A 42 (2009), no. 40, 404015.

[61] M. Skopenkov, H. Pottmann, P. Grohs, Ruled Laguerre minimal surfaces, Math. Z. 272 (2012), 645-674.

[62] Y.-P. Song, Laguerre isothermic surfaces in $\mathbb{R}^{3}$ and their Darboux transformation, Sci. China Math. 56 (2013), 67-78.

[63] A. Szereszewski, L-isothermic and L-minimal surfaces, J. Phys. A 42 (2009), no. 11, 115203.

[64] G. Thomsen, Über konforme Geometrie I: Grundlagen der konformen Flächentheorie, Hamb. Math. Abh. 3 (1923), 31-56.

[65] M. Umehara, K. Yamada, A parametrization of the Weierstrass formulae and perturbation of complete minimal surfaces in $\mathbb{R}^{3}$ into the hyperbolic 3-space, J. Reine Angew. Math. 432 (1992), 93-116.

[66] K. Voss, Verallgemeinerte Willmore-Flächen, Mathematisches Forschungsinstitut Oberwolfach, Workshop Report 42 (1985), 22-23. 
